\title{
ALCUNI PASSI DEL POEMA DI GILGAMESH IN DUE TAVOLETTE ITTITE INEDITE*
}

\author{
Nota del s.c. CLELIA MORA (*) e MAURO GIORGIERI (**)
}

(Adunanza del 20 ottobre 2016)

SuNTO. - Lo studio di due frammenti di tavoletta in scrittura cuneiforme contenenti alcuni passi della versione ittita della saga di Gilgamesh offre l'occasione per presentare e approfondire alcuni aspetti relativi alla riscoperta, all'interpretazione e alla fortuna di questo poema che ebbe una larga diffusione nel Vicino Oriente antico. Ad una prima parte in cui vengono trattate queste tematiche di carattere generale, segue una sezione dedicata all'esame e alla lettura dei nuovi frammenti e al loro interessante apporto dal punto di vista contenutistico.

$$
* * *
$$

ABSTRACT. - In this paper we present two fragments of a cuneiform tablet that contain some passages of the Gilgamesh saga in the Hittite language. They give us the opportunity to delve into some aspects concerning the rediscovery, the interpretation and the fortune of this poem widely widespread throughout the Ancient Near East. The first part of the paper deals with these general issues, while the second one focuses on the philological examination of the new fragments and the interesting contribution they offer from the content viewpoint.

(") Università degli Studi di Pavia, Italy. E-mail: clelia.mora@unipv.it

(**) Università degli Studi di Pavia, Italy. E-mail: mauro.giorgieri@unipv.it

\# Il lavoro è frutto di una ricerca in comune dei due autori. In particolare i $\$ \mathbb{S}$ 1-5 sono a cura di C. Mora, i $\$ \$$ 6-7 a cura di M. Giorgieri. 


\section{INTRODUZIONE}

La presentazione di due interessanti frammenti di tavolette in scrittura cuneiforme ci offre l'occasione per un approfondimento su una delle testimonianze più importanti della letteratura del Vicino Oriente antico, il poema di Gilgamesh.

I due preziosi reperti sono conservati da un privato che, con il permesso di pubblicazione, ci ha gentilmente fornito le fotografie. Si tratta di due frammenti di tavolette in scrittura cuneiforme provenienti presumibilmente dal sito dell'antica capitale degli Ittiti, Hattusa, nell'Anatolia centro-settentrionale (Fig. 1).

Il sito della capitale ittita, scoperta ai primi del '900 da una missione archeologica tedesca, ${ }^{1}$ è infatti il bacino principale di provenienza delle tavolette cuneiformi in lingua ittita.

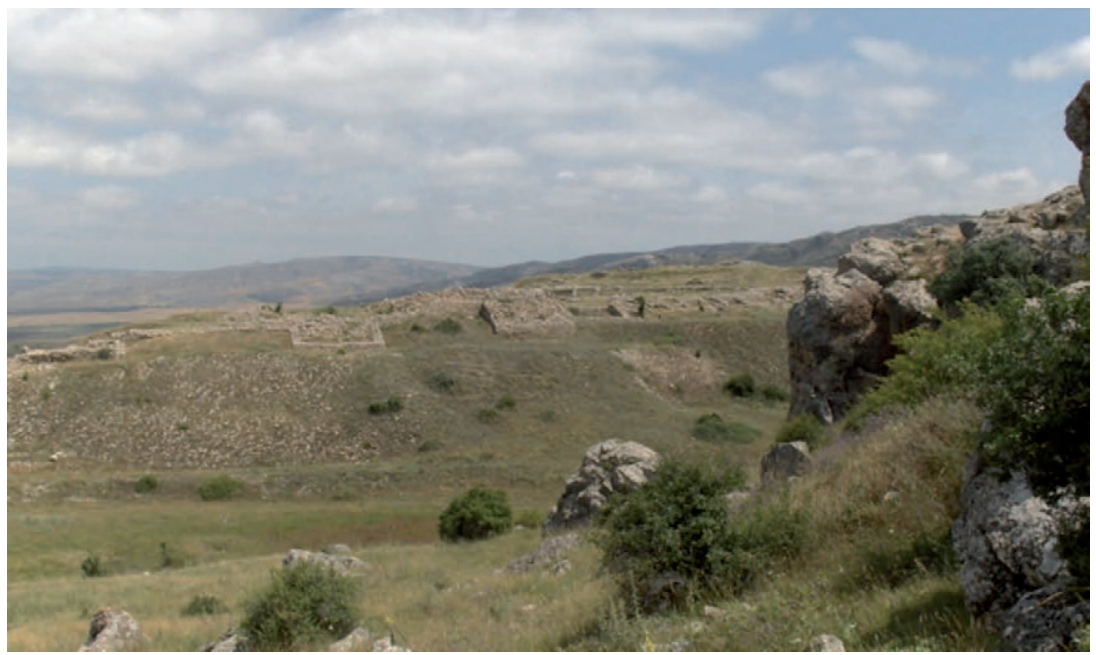

Fig. 1 - Tratto di mura della capitale ittita Hattusa (foto C. Mora).

Il dominio del regno ittita si impose in Anatolia a partire dal XVII secolo a.C. e, con alterne vicende, si protrasse fino agli inizi del XII secolo a.C., quando quello che era ormai diventato un 'impero' sovra-

1 Gli scavi, diretti da Hugo Winckler, iniziarono nel 1906; per la storia e la descrizione delle scoperte cfr. in particolare Schachner 2011. 
regionale, esteso anche al di fuori dell'Anatolia, si disgregò e dissolse per motivi e in circostanze non ancora del tutto chiariti (Fig. 2).

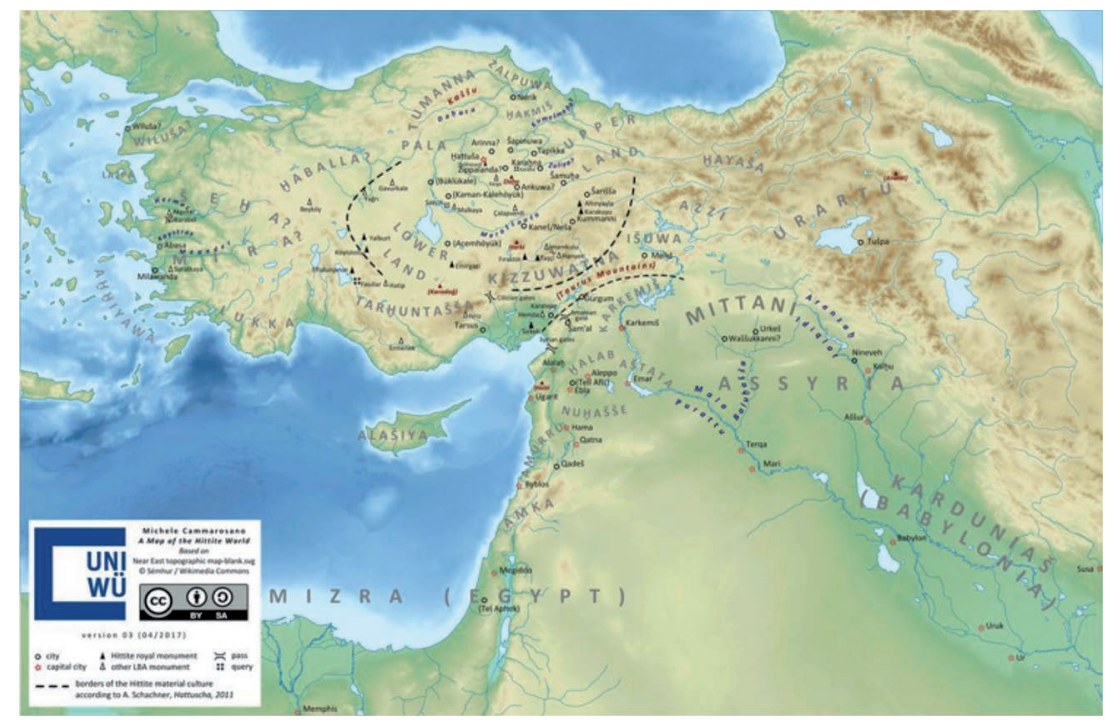

Fig. 2 - Carta del Vicino Oriente all'epoca degli Ittiti (a cura di M. Cammarosano)).

Gli Ittiti, popolazione di lingua indoeuropea sulle cui origini e luogo di provenienza - prima del loro insediamento in Anatolia - ancora si discute, stabilirono la prima sede del regno nella parte centro-settentrionale della penisola anatolica e acquisirono dalle regioni vicine la scrittura cuneiforme, già in uso da molto più di un millennio in Mesopotamia.

La scrittura cuneiforme ebbe origine infatti nella seconda metà del IV millennio a.C. nella Mesopotamia meridionale, allora abitata dai Sumeri; si tratta di un sistema di scrittura molto complesso che utilizza segni con valore fonetico-sillabico e segni con valore ideografico-logografico; i segni erano tracciati con uno stilo su 'tavolette' di argilla ancora tenera che poi venivano lasciate seccare al sole. ${ }^{2}$ I segni si stilizzarono

2 In una terra estremamente povera di materie prime come la Mesopotamia meridionale, l'argilla era un materiale abbondante e onnipresente: oltre che come supporto privilegiato per la scrittura, l'argilla, in forma di mattoni cotti al sole, era utilizzata per ogni tipo di costruzione; i miti più antichi narrano inoltre che con l'argilla gli dei avevano preparato il modello per la creazione dell'uomo. 
sempre più nel corso del tempo, fino ad assumere la forma di piccoli 'chiodi / cunei', da cui la denominazione moderna. ${ }^{3}$ Nel corso del III millennio la scrittura fu acquisita anche dagli Akkadi, popolazione di lingua semitica insediata in quell'epoca in Mesopotamia. ${ }^{4}$ Dalla Mesopotamia la scrittura si diffuse, a partire dalla metà del III millennio a.C., ad altre regioni vicine, come la Siria e l'Anatolia.

Le tavolette qui presentate sono di particolare interesse, perché, in base ad un attento esame, è stato possibile riconoscere la loro appartenenza ad una versione ittita del poema di Gilgamesh (si veda più avanti, $\$ 7.1-2)$ di cui riportano alcuni brani fino ad ora non documentati.

La saga di Gilgamesh è uno dei più importanti testi dell'antichità: è nota e famosa per le vicende che racconta, per le figure emblematiche di Gilgamesh e di Enkidu, per la simbologia sottesa ad ogni personaggio, umano o divino, e ad ognuna delle imprese narrate. E' un testo a carattere epico, letterario, religioso che è stato avvicinato, per la sua complessità, all'Iliade e all'Odissea, ma li precede sicuramente di molte centinaia di anni.

\section{LA SAGA DI GILGAMESH NEL CONTESTO DELLA LETTERATURA ANTICO-ORIENTALE E LE SUE DIVERSE REDAZIONI}

Nell'ambito della documentazione scritta del Vicino Oriente antico non è facile distinguere testi di carattere letterario da testi utilizzati per la pratica e la devozione religiosa. Le preghiere, gli inni alle divinità e ovviamente i testi mitologici spesso si avvicinano, per aspetti narrativi e /o formali, alle caratteristiche di quello che per noi è un testo letterario. Si tratta in realtà quasi sempre di documenti che si rifanno a modelli della tradizione, e connessi ad un utilizzo di tipo pratico, ad esempio una celebrazione o una recitazione in occasione dello svolgimento di un rito, oppure per ottenere perdono dagli dèi, per lo scioglimento di un vincolo o un incantesimo, per l'allontanamento di un presagio negativo. Il racconto

3 La definizione 'cuneiforme' sembra essere stata coniata indipendentemente, tra fine XVII secolo-inizio XVIII, da Thomas Hyde, professore di Ebraico a Oxford, e da Engelbert Kämpfer, naturalista e viaggiatore (Persia, estremo Oriente).

4 Successivamente la lingua accadica divenne la lingua più diffusa nel Vicino Oriente e il mezzo di comunicazione per gli scambi diplomatici. 
vero e proprio si trova quindi frequentemente inserito (e talvolta 'sacrificato') entro strutture non tipicamente letterarie, almeno secondo il nostro metro di giudizio. In alcuni testi le parti narrative sembrano più libere da schemi e da vincoli precostituiti: è il caso di alcune leggende e racconti della regione levantina, ${ }^{5}$ di alcuni famosi testi egiziani, ma soprattutto del poema che narra la storia di Gilgamesh, che ha superato i confini spaziali della zona d'origine, la Mesopotamia meridionale. Le prime testimonianze di poemi epici sulle imprese di Gilgamesh risalgono probabilmente alla fine del III millennio a.C.; si tratta di composizioni redatte in lingua sumerica e provenienti dai centri della Mesopotamia meridionale, dove sono state ritrovate nel corso di scavi archeologici nei primi anni del '900. ${ }^{6}$ Alcune di queste storie sono poi confluite in quello che viene considerato il testo canonico del poema, ritrovato in diverse copie nella cosiddetta 'biblioteca' di Assurbanipal a Ninive (VII sec. a.C.), ma la cui redazione risale con tutta probabilità ad alcuni secoli prima, probabilmente al periodo di regno del re babilonese Nabucodonosor I (fine del XII sec. a.C.). Un primo nucleo di un racconto organico delle imprese di Gilgamesh in lingua accadica, intitolato Šütur eli šarrī («Colui che eccelle sopra i re»), certamente più breve rispetto al testo 'classico', risale all'epoca paleobabilonese (prima metà del II millennio a.C.).?

Dagli archivi della capitale ittita provengono versioni del poema in lingua accadica, in lingua ittita e anche in lingua hurrita. ${ }^{8} \mathrm{I}$ frequenti

5 Ugarit e zona fenicia, tra Tardo Bronzo e prima Età del Ferro (cioè tra seconda metà del II millennio a.C. e inizio del I millennnio a.C.).

6 Cfr. Falkenstein 1968; George 2003, 4 ss.; si veda anche Pettinato 1992, 110 ss. $\mathrm{Si}$ tratta di composizioni che narrano singole imprese dell'eroe, in genere considerate a sé stanti, non collegate in un ciclo unitario (ma si veda il recente riesame in Gadotti 2014, e precedentemente Sallaberger 2013, 59 ss., Mittermeyer 2010, 7). Per l'ipotesi che esistesse anche all'epoca del regno di Akkad (seconda metà III millennio a.C.) un poema organico su Gilgamesh in lingua accadica, si veda Buccellati, in stampa (v. più avanti, $\mathbb{S} 4$ ). Per la possibilità di una rappresentazione dell'eroe su sigilli di epoca accadica e per testi da Ebla che potrebbero fare riferimento a episodi della saga cfr. Frayne 2010.

7 Cfr. George 2003, 22 ss. L'epoca paleobabilonese è nota in particolare per il regno del re Hammurabi, grande conquistatore e autore della famosa raccolta di leggi che porta il suo nome. Per l'evoluzione del poema cfr. Tigay 1982; Wasserman 2011, 2; Sallaberger 2013, 80 ss.

8 Popolazioni di lingua hurrita sono attestate nella zona settentrionale della Mesopotamia nell'Età del Bronzo, tra III e II millennio a.C. Per queste versioni provenienti dalla capitale degli Ittiti Hattusa si veda più avanti, $\mathbb{\$} 6$. 
ritrovamenti di parti della saga in regioni esterne alla Mesopotamia ${ }^{9}$ testimoniano dunque della diffusione e notorietà del testo presso gli ambiti scribali e colti di tutto il Vicino Oriente tra secondo e primo millennio a.C.

Dalla documentazione antica conserviamo alcune indicazioni relative al nome del dotto scriba che aveva redatto la versione canonica: Sin-leqi-unninni. Si tratta di un caso quasi unico nell'ambito della tradizione antico-orientale, che di norma non contempla 'autori', ma soltanto esperti copisti. ${ }^{10}$ Questa versione canonica del poema si compone di 12 tavole. $^{11}$

A queste versioni scritte, colte e canoniche, si affiancava molto probabilmente una tradizione orale, che ad esse attingeva forse introducendo anche varianti e innovazioni locali e 'popolari'. Riportiamo in proposito un commento di F. D’Agostino: ${ }^{12}$

A questa enorme diffusione di Gilgameš ${ }^{13}$ avrà però senz'altro contribuito anche il fascino fortissimo che il racconto in sé, con le avventure così umane $\mathrm{e}$ universali che coinvolgono il sovrano di Uruk, promana a chi lo ascolta. Immaginiamo come esso venisse raccontato da narratori professionisti nelle lunghe pause dei viaggi in carovane commerciali, note già dal III millennio a.C., allorché le avventure complicate e meravigliose dell'eroe Gilgameš servivano a distrarre gli affaticati viandanti dopo un giorno di cammino. È stato notato, d'altro canto, come temi narrativi presenti in questo poema si ritrovino poi anche nel mondo greco (nell'Odissea addirittura) e nella grande letteratura fantastica araba (nel Racconto di Buluqiya de Le mille e una notte). Storie così straordina-

9 Oltre ai ritrovamenti in area anatolica vanno ricordati anche frammenti di una versione mediobabilonese ritrovati a Megiddo in Palestina e a Emar e Ugarit, in Siria (cfr. D’Agostino 2017, 62 ss., con riferimenti a Goetze - Levy 1959, Bottéro 1992, 272 ss. e Pettinato 1992, 108 ss. per le testimonianze da Megiddo; Arnaud 1987, nn. 781-782 per quelle da Emar; per le testimonianze da Ugarit si veda George 2007). Per l'influenza dell'epopea di Gilgamesh sulla letteratura più tarda della zona levantina cfr. Steymans 2010.

10 Sulla figura di Sin-leqi-unninni cfr. George 2007, 28 ss.; Arnaud 2007; D’Agostino, 2017, 68 ss.

11 Si veda più sotto, $\$ 3.1$ sul problema della supposta incongruità della XII tavola. Cfr. inoltre George 2003, 31 ss. sulla questione della corrispondenza o meno del testo attribuito a Sin-leqi-unninni e quello della versione canonica.

12 Cfr. D’Agostino 2017, 62-63, con rimando a Dalley 1991 e a George 2003.

13 L'Autore si riferisce alla diffusione delle versioni scritte del poema. 
rie divennero, attraverso il racconto orale di professionisti della parola, patrimonio comune di una enorme quantità di differenti popolazioni, che rielaborarono il racconto, ripensarono il personaggio e il suo significato per la propria cultura e lo tramandarono ai posteri.

\section{LA VERSIONE 'CANONICA' DEL POEMA}

\subsection{Contenuto (tavolette I-XI)}

La prima tavoletta si apre con la presentazione di Gilgamesh, re di Uruk, per due terzi dio e per un terzo uomo: Gilgamesh è figlio di una dea e di un re; l'eroe viene presentato nell'incipit del testo come "colui che vide l'abisso, il fondamento della terra e di tutto fu esperto" ${ }^{14}$ seguono la presentazione di Enkidu, un essere selvaggio non civilizzato, creato dagli dèi come alter ego di Gilgamesh per porre un freno alla sua arroganza, e l'incontro tra Gilgamesh ed Enkidu; dopo uno scontro e una lotta violenta, nasce tra i due una profonda amicizia; la narrazione prosegue (tavola IV ss.) con le avventure dei due eroi, che iniziano con la spedizione alla 'Foresta dei cedri' per sconfiggere il mostro Humbaba; l'ingresso nella foresta e l'incontro con il mostro sono preceduti da una serie di sogni premonitori. La foresta è descritta come un luogo enorme che incute terrore per l'altezza e l'intrico delle piante e per il rumore incessante. Dopo una lotta terribile, il mostro/demone Humbaba, guardiano della foresta, viene ucciso. I due eroi tagliano gli alberi della foresta, anche per farne offerte agli dèi, e ritornano in patria; la parte che segue è dedicata ai tentativi della dea Ištar di sedurre Gilgamesh, che non si lascia lusingare e oppone un netto rifiuto, anche perché i precedenti amanti non hanno avuto un destino felice. La dea quindi gli scatena contro il «Toro Celeste». ${ }^{15}$ Con

14 D'Agostino 2017, 76; si veda anche l'ediz. critica di George 2003, 539: «[He who saw the Deep, the] foundation of the country, [who knew ....] was wise in everything!». L'incipit della versione canonica in dodici tavole differiva dunque da quello della versione paleobabilonese (v. sopra).

15 V. D’Agostino 2017, 132, per una possibile simbologia di questa figura: «Il Toro Celeste (letteralmente 'del cielo') rappresenta nell'immaginario mitico dei Sumeri e dei Babilonesi il caldo torrido dell'estate che secca i pozzi e il fiume Eufrate e uccide l'erba sui pascoli». 
fatica Gilgamesh ed Enkidu riescono ad avere la meglio sul Toro, che viene ucciso; la città di Uruk festeggia i due eroi e il mancato pericolo. $\mathrm{Ne}$ consegue l'ovvia ira della dea, ed Enkidu durante la notte ha un brutto sogno; nella tavola VII si trova il racconto del sogno, conservato con maggiori dettagli nella versione ittita della saga, che descrive una riunione delle maggiori divinità che condannano a morte uno dei due amici, resisi colpevoli di imprese contrarie al volere degli dèi. Il predestinato è Enkidu, che dapprima è preda di deliri e si lascia andare ad una serie di invettive, poi, placato e indebolito, si rassegna alla morte che lo raggiunge inesorabile, gettando Gilgamesh nella disperazione più profonda. Alla morte dell'amico, Gilgamesh recita una lunga litania, alla quale segue la cerimonia funebre.

Dopo il racconto della morte di Enkidu si apre la seconda parte del poema (tavole VIII-XI), incentrato sulla ricerca dell'immortalità da parte di Gilgamesh. Affranto dal dolore, l'eroe decide di recarsi da Utanapishtim, l'uomo sopravvissuto al diluvio, che ha conquistato così la vita eterna. Ma il percorso per raggiungere il luogo dove risiede Utanapishtim è lungo e irto di pericoli. Gilgamesh affronta una serie di avventure, di incontri fantastici e terribili, di percorsi difficilissimi (ricordiamo in particolare l'incontro con gli uomini-scorpione, attendenti del dio Sole, e il lungo viaggio nelle buie viscere della montagna per raggiungere il giardino incantato del Sole). Segue l'incontro con la 'taverniera' Siduri, ricco di dialoghi e di riflessioni profonde; si ritorna tuttavia presto al tema centrale e la stessa Siduri, dapprima scettica, indica all'eroe il nome di un traghettatore, Ur-Shanabi, che può condurlo da Uta-napishtim. Dopo una serie di altre mirabolanti avventure, finalmente Gilgamesh raggiunge la sua meta. Segue quindi un dialogo non incoraggiante con il sopravvissuto al diluvio, in cui il vecchio saggio cerca di convincere Gilgamesh dell'ineluttabilità della condizione umana; dopo le insistenze del giovane eroe, inizia il racconto del diluvio: gli dèi decidono di provocare un diluvio che distruggerà tutta l'umanità ${ }^{16}$ in segreto, Uta-napishtim viene informato della decisione e potrà salvarsi costruendo una nave capace di resistere alle acque, e che salverà anche tutti gli animali esistenti. Il racconto dell'inizio della tem-

16 La decisione non è motivata nel racconto del poema, ma da altri miti anticomesopotamici, primo fra tutti il mito di Atram-hasis, il "super-saggio", conosciamo le discussioni tra gli dei e le motivazioni che hanno portato a questa decisione. 
pesta è potente e terribile («Il silenzio di Adad - il dio della tempesta si impadronì del cielo, e fece diventare tenebra tutto ciò che luceva»), ${ }^{17}$ e prosegue con la narrazione dei venti impetuosi e delle piogge che provocano tremende devastazioni; dopo alcuni giorni, in un momento di calma, Uta-napishtim libera degli uccelli per accertarsi che sia tutto finito e finalmente esce a contemplare un paesaggio di morte e di silenzio.

Alla fine, dopo alcune prove a cui è ancora sottoposto, Gilgamesh si appresta a tornare, accompagnato dal traghettatore. Seguendo le indicazioni di Uta-napishtim, raccoglie in fondo al mare la pianta della giovinezza ma, mentre si immerge in una pozza d'acqua fresca per detergersi, la pianta gli viene sottratta da un serpente che immediatamente ringiovanisce mutando la pelle. A Gilgamesh non resta che far ritorno a Uruk, meno baldanzoso ma più saggio e più 'umano'. La tavola XI si conclude con alcune righe che, riallacciandosi al prologo, descrivono l'imponenza e la bellezza della città.

\subsection{La tavoletta XII}

Dopo questa parte che a noi appare sotto tutti gli aspetti come la conclusione del racconto, si trova tuttavia un'ulteriore tavoletta (la XII), che secondo alcuni studiosi potrebbe essere stata aggiunta alla versione classica in un momento successivo non facilmente precisabile. Non si conoscono i motivi di questo prolungamento; in effetti questa tavoletta tratta di un tema non del tutto connesso con la parte finale della saga come presentata nella tavoletta XI. Nella XII tavoletta viene ripresa, tradotta in lingua accadica, una parte di un precedente poema sumerico (Bilgames, Enkidu e gli Inferi), in cui ritorna protagonista Enkidu, che si reca negli Inferi per recuperare alcuni oggetti di Gilgamesh caduti in un buco del terreno. Enkidu, nonostante le raccomandazioni di Gilgamesh, rimane prigioniero del regno dei morti, e non riesce a ritornare. Gilgamesh invoca l'aiuto degli dèi, che concedono alla parvenza di Enkidu di riapparire attraverso una fessura. Gilgamesh gli chiede allora di raccontargli la sua esperienza:

Dimmi amico mio, dimmi amico mio, dimmi gli ordinamenti degli Inferi che tu hai visto

17 Trad. D’Agostino 2017, 178. 
Io non te li posso dire, amico mio, non te li posso dire! Se io infatti ti dicessi gli ordinamenti degli Inferi che ho visto, allora tu ti butteresti giù e piangeresti. ${ }^{18}$

Ne segue il resoconto, tristissimo e cupo, della sorte dell'uomo nell'aldilà, che riflette le credenze degli antichi mesopotamici sull'argomento. Tra le molteplici ipotesi avanzate per giustificare la presenza di questo racconto dopo quella che appariva a tutti gli effetti la parte finale, ci pare interessante la proposta di George (2003, 52 ss.), che pensa ad una ripresa del motivo della morte di Enkidu per ragioni cultuali, collegate all'istituzione di riti funebri. ${ }^{19}$ La citazione di un passo (George 2003, 54) può chiarire meglio la sua proposta di interpretazione:

The epic's central concern with death and its lesson that no man can live for ever, not even the greatest of heroes and wises of kings, are eminently appropriate to occasions on which the living bury the dead, commemorate their names and succour their ghosts. If the epic came eventually to have a function in such a context, Tablet XII would still be an appendage, but not an idle one; as a postscript to the great poem it would form an eloquent reminder of the duties owed by men to their ancestral spirits.

\subsection{La riscoperta delle tavolette contenenti la narrazione del poema}

Come è stato riscoperto il poema di Gilgamesh? Da quando è stato possibile per noi moderni rileggerlo e confrontarci con un testo così ricco di avventure e così profondamente umano e universale nei sentimenti che trasmette?

Negli anni '40 dell' 800 il diplomatico francese P.E. Botta ${ }^{20}$ e, poco dopo, l'inglese H.A. Layard, diedero inizio ai primi scavi archeologici in Mesopotamia settentrionale (nord dell'attuale Iraq), riscoprendo i resti delle antiche capitali assire Dur Sharrukin, Kalhu e Ninive. Gran parte del materiale scoperto veniva poi trasportato, con grandi fatiche

18 Trad. Pettinato 1992, 235.

19 Sulle diverse interpretazioni proposte si rimanda a George 2003, 47 ss.: Tablet XII: What, When and Why?

20 Botta svolgeva la funzione di vice-console a Mosul: allora gran parte dei territori del Vicino Oriente erano sotto il dominio dell'Impero ottomano. 
e molti mesi di viaggio, nei grandi musei di Londra e di Parigi. ${ }^{21}$ Tra questi reperti, le migliaia di tavolette scritte in cuneiforme suscitarono enorme interesse tra gli studiosi che da alcuni decenni si stavano cimentando nell'arduo tentativo di decifrare la scrittura. ${ }^{22} \mathrm{Nel} 1857$, in seguito ad una iniziativa della Royal Asiatic Society di Londra, fu proclamato l'avvenuto deciframento della scrittura cuneiforme e della lingua accadica, la più diffusa nell'antico Oriente. Pochi anni dopo George Smith, un giovane interessato allo studio di questa scrittura e dei relativi documenti, fu ammesso allo spoglio delle tavolette nei magazzini del British Museum. Nel 1872 Smith, nel corso di una conferenza, annunciò di aver identificato tra le migliaia di tavolette esaminate un racconto del diluvio per molti aspetti simile a quello presente nella Bibbia. Dopo altre ricerche lo stesso Smith riconobbe l'appartenenza dei frammenti al poema di Gilgamesh e fu inviato in terra assira proprio con lo scopo di individuare, tra i resti dei palazzi appena scoperti, altre tavolette che potessero aggiungere conferme alla diffusione del racconto del diluvio prima della redazione del testo biblico. Smith scoprì in effetti altre tavolette con racconti analoghi e le attribuì al poema di Gilgamesh, ma oggi sappiamo che si trattava in realtà di parti di un importante mito di fondazione mesopotamico, quello di Atram-Hasis. ${ }^{23}$

La scoperta delle tavolette contenenti il racconto del diluvio inserito nel poema di Gilgamesh ha rappresentato uno degli eventi più importanti e famosi nella storia della riscoperta delle grandi civiltà del Vicino Oriente antico, e ha avuto grande ripercussione presso un pubblico molto vasto, non soltanto nell'ambito degli studi specialistici. ${ }^{24}$

Dopo la fase avventurosa ed 'eroica' delle prime scoperte, nuove

21 Lo scavo del canale di Suez fu avviato solo dopo alcuni anni (l'inaugurazione avvenne nel 1869), e quindi era ancora necessario circumnavigare l'Africa per raggiungere i porti europei partendo dal Golfo Persico.

22 Copie di testi in scrittura cuneiforme erano giunte in occidente già dal ' 600 , portate da alcuni viaggiatori / esploratori che si recavano nelle regioni del Vicino Oriente.

23 Per la storia della scoperta delle prime tavolette appartenenti al poema di Gilgamesh cfr. in particolare S.M. Chiodi in Pettinato 1992, 82 ss. e D'Agostino 2017, 27 ss. Per le diverse versioni del testo e la loro composizione cfr. George 2003, 1 ss. (Introduction) e 159 ss. (Part Two).

24 Si veda ad es. il risalto dato recentemente a questa scoperta in un fascicolo di National Geographic, Storica (agosto 2017, 108 ss.), con un ampio e documentato articolo: George Smith trova la tavoletta del Diluvio Universale. 
tavolette o frammenti di tavoletta si vanno ad aggiungere ancora oggi all'insieme del testo, che è quindi in costante ricomposizione. Un esempio recente è rappresentato dal ritrovamento, abbastanza casuale nelle sue modalità, di alcune parti della Tavola $\mathrm{V}$, che aggiungono nuovi particolari all'avventura dei due eroi nella Foresta dei Cedri. ${ }^{25}$ In fondo, anche questo nostro piccolo contributo aggiunge ora qualcosa di nuovo all'insieme.

Il fatto di poter attingere ai testi originali, e di poterne confrontare diverse redazioni, databili a periodi diversi nella storia dell'antica Mesopotamia, pone la saga di Gilgamesh in una situazione certamente privilegiata per quanto riguarda gli studi sull'origine, sulla storia e sulla ricostruzione del testo. Come dice Pettinato: ${ }^{26}$

I classicisti dibattono ancor oggi sull'unitarietà o meno dell'Iliade e dell'Odissea, e scandagliano il testo verso dopo verso per scoprirne le fonti. Noi, al contrario, ci troviamo, con l'epica di Gilgamesh, in una situazione veramente ottimale. Non abbiamo bisogno di sezionare il testo per scoprire eventuali fonti più antiche dell'Epopea classica.

\section{LA FORTUNA DEL POEMA NELL'ANTICHITÀ. \\ LE TESTIMONIANZE ARTISTICHE}

Nel $₫ 2$ abbiamo accennato alle numerose versioni del poema ritrovate in diverse zone del Vicino Oriente; anche se queste testimonianze a noi pervenute, pur numerose, rappresentano probabilmente solo una piccola parte delle tradizioni (e traduzioni) che esistevano nell'antichità, sono tuttavia sufficienti a darci un'indicazione della diffusione e dell'immensa fortuna di cui ha goduto questa 'storia' esemplare, nelle sue infinite varianti. La figura di Gilgamesh appare inoltre, come sovrano, dio, semi-dio o eroe, in altre tipologie di documenti, che hanno tra l'altro portato a discutere della 'storicità' del personaggio (in genere negata da gran parte degli studiosi). Tra questi documenti si ricordano, a titolo di esempio, La Lista reale sumerica, in cui Gilgamesh è citato come un re divinizzato «figlio di uno sconosciuto», ${ }^{27}$ il cosiddet-

25 Cfr. il recente articolo di Al-Rawi - George 2014.

26 Pettinato 1992, 11, con riferimento a Cooper 1981.

27 Cfr. Pettinato 1991, 137 e 143 ss., in cui si ricordano gli antichi e leggendari sovrani della Mesopotamia tramandati da autori antichi (ad es. Eliano, II-III sec. d.C.). 
to Inno di Shulgi (sovrano della III dinastia di Ur, della fine del terzo millennio a.C., che si definisce «fratello di Gilgamesh»), fino ad un documento ritrovato a Sultantepe, in Turchia: $:^{28}$ si tratta di un testo, in forma di lettera, in cui Gilgamesh chiede ad un altro sovrano una grande quantità di lapislazzuli per costruire una statua in ricordo dell'amico Enkidu (con chiaro riferimento all'episodio della morte del compagno di avventure). ${ }^{29}$ Passando ad altri generi testuali, il nome di Gilgamesh ricorre anche in alcuni testi oracolari che ne rievocano le gesta eroiche, e anche in testi di esorcismo. ${ }^{30}$

Riguardo ai possibili influssi sulla letteratura non cuneiforme dell'antichità, ad es. sui poemi omerici, ma anche su alcune storie tramandate dai testi di Qumran, da Luciano, dalle Mille e una notte (testimonianze sulle quali molto si è discusso) rimandiamo alla dettagliata e documentata trattazione di George (2003, 54 ss.: The Epic of Gilgameš outside the Cuneiform Tradition).

Si è anche discusso, tra gli studiosi, sulle possibili rappresentazioni di episodi o di personaggi della saga di Gilgamesh in opere artistiche o prodotti di artigianato coevi ai periodi di elaborazione e diffusione della saga: ${ }^{31}$ molti reperti provenienti dagli scavi archeologici nei luoghi in cui il poema aveva diffusione nell'antichità, in particolare sigilli e placchette, sono stati indagati con questo intento. Le difficoltà principali per una sicura identificazione delle immagini con i personaggi o $i$ temi della saga sono dovute sia alla mancanza di riferimenti scritti a descrizione delle rappresentazioni artistiche, sia alla mancanza di elementi o dettagli specifici che permettessero l'identificazione sicura. ${ }^{32}$ Inoltre, la quantità di materiale, scritto $o$ artistico, a noi pervenuto rappresenta soltanto una minima parte di quello sicuramente disponibile e

Per questo passo della Lista reale sumerica, dove si menziona il padre di Gilgamesh, v. anche - con altre ipotesi di traduzione - Wilcke 1989, 562 e Glassner 2004, 121.

28 Probabilmente si tratta di un testo di tipo scolastico; si veda l'edizione in Gurney et al. 1957; cfr. anche Kraus 1980, 109-121.

29 Sulla vastità della documentazione antica relativa a Gilgamesh si vedano le osservazioni di Pettinato (1992), in particolare 74 ss., e di D’Agostino 2017, 205 ss.

30 V. Pettinato 1992, 77, con riferimenti; George 2003, 132 ss.

31 Per una raccolta di studi dedicata proprio all'iconografia di Gilgamesh cfr. la pubblicazione di Steymans Hrsg. 2010.

32 Questo tipo di problemi riguarda gran parte della produzione artistica antico-orientale: cfr. in proposito le osservazioni di Lambert 1987. 
circolante nell'antichità: questo dato riduce ulteriormente le possibilità di trovare corrispondenze sicure tra i due ambiti. ${ }^{33}$

Come già accennato, un esame dettagliato delle diverse tipologie di reperti e di rappresentazioni e una critica a metodologie di ricerca poco accurate si trova in uno studio di Lambert del $1987::^{34}$ nella seconda parte del lavoro di Lambert sono selezionati alcuni reperti che possono superare un'analisi filologica accurata (sia dal punto di vista testuale che della rappresentazione artistica). Nel più recente contributo di Kelly-Buccellati (2006) si propone, alla luce di un'analisi approfondita e di criteri particolarmente rigorosi, di riconoscere la rappresentazione dei due eroi Gilgamesh e Enkidu in una placchetta di calcare ritrovata a Urkesh (Siria settentrionale, area hurrita) e risalente alla seconda metà del III millennio a.C. (Fig. 3).
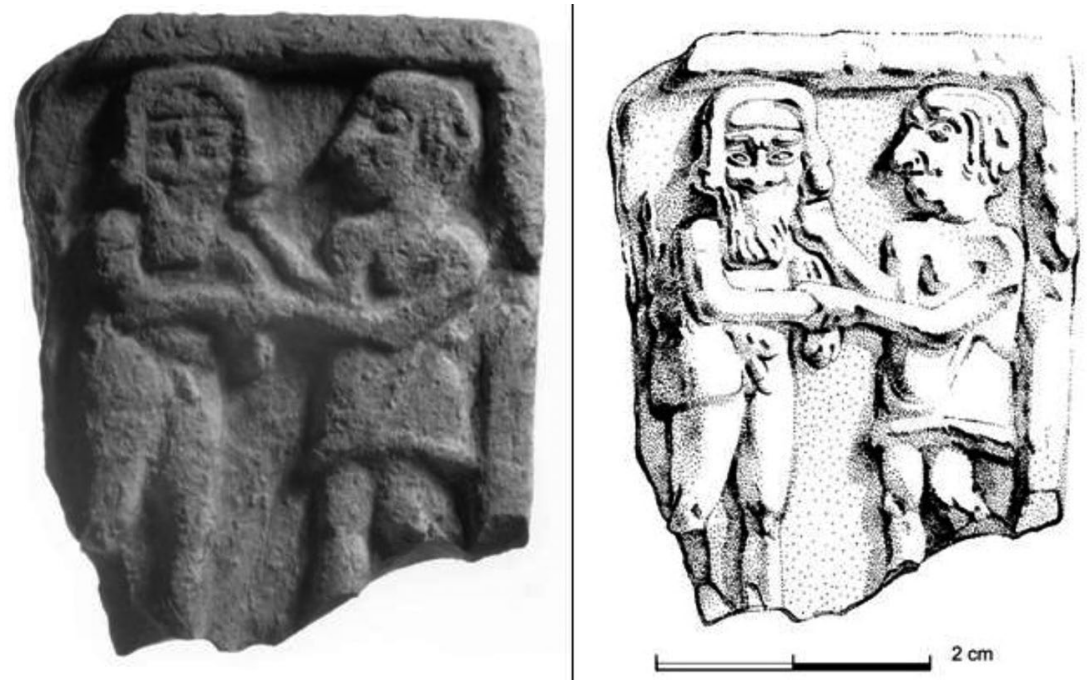

Fig. 3 - Placchetta da Urkesh (da http://www.urkesh.org).

Questo importante documento, che molto probabilmente rappresenta la parte superiore destra di una più ampia rappresentazione in quattro 'quadri', è utilizzato da G. Buccellati, in collegamento con altri

33 Tra i molti studi che discutono di questa problematica cfr. Matthiae 1992.

34 Cfr. poco sopra, nota 32. 
indizi, a supporto dell'ipotesi dell'esistenza di un testo epico unitario relativo a Gilgamesh già all'epoca della dinastia di Akkad (cfr. anche poco sopra, $\$ 2) .^{35}$

\section{LA FORTUNA IN EPOCA CONTEMPORANEA}

Come per tutta la letteratura e i testi scritti dell'antico Oriente in generale, anche dell'epopea di Gilgamesh si persero le tracce già nell'antichità, almeno per quanto riguarda la tradizione occidentale. Soltanto con la scoperta, nella seconda metà dell'800, dei primi frammenti di tavoletta che raccontavano la storia del diluvio (si veda sopra, $\$ 3.3$ ), le imprese dell'eroe mesopotamico ritornarono prepotentemente 'attuali'. Tra le edizioni più importanti del testo nei primi decenni del '900 ricordiamo quella di A. Ungnad, Das Gilgamesch-Epos (Ungnad 1911) e quella fondamentale di R.C. Thompson, The Epic of Gilgamish (Thompson 1930). ${ }^{36}$ Le prime edizioni del testo e gli studi scientifici furono accompagnati da dibattiti, discussioni e articoli di giornali che interessarono spesso anche un pubblico meno colto e specializzato (anche, o forse soprattutto, per le analogie con alcuni importanti passi biblici, come si è detto).

Anche alcuni famosi intellettuali e letterati del ' 900 si interessarono alle vicende dell'eroe mesopotamico e vi fecero cenno nelle loro opere o nei loro scritti. Ricordiamo tra gli altri R.M. Rilke, che in alcune lettere riferisce di aver letto con grandissimo interesse la traduzione del poema, dichiarandosi colpito dalla figura di Gilgamesh e dalle vicende di cui è protagonista. In uno studio dedicato all'esame di queste testimonianze, l'assiriologo W.L. Moran mette l'accento sull'atteggiamento

35 L'ipotesi di Buccellati (v. Buccellati, in stampa) è fondata, oltre che sul ritrovamento della placchetta di Urkesh databile ad un'epoca precedente al periodo paleobabilonese, su altri interessanti indizi, quali la menzione di Ebla in un testo paleobabilonese di recente pubblicazione (OB Schøyen 2 26, v. George 2003, 234 s.); ma in particolare, Buccellati sottolinea come l'ideologia della saga unitaria di Gilgamesh composta in epoca paleobabilonese corrisponda, a partire dal 'titolo' («Colui che eccelle sopra i re»), all'ideale eroico e alle ambizioni di espansione e di conquista che caratterizzavano la dinastia di Akkad.

36 Per una rassegna delle prime edizioni e dei primi studi cfr. D’Agostino 2017, 51 ss 
romantico del poeta nei confronti di un'opera così antica, che «raised in the mists of the dawn of history, now destroyed or surviving only in fragments, with the power and pathos that attach to remnants of the ancient past». ${ }^{37}$

Numerosi sono gli studi sull'interesse manifestato nei confronti del poema di Gilgamesh da altri grandi autori tedeschi che lo citano in alcune lettere, quali Thomas Mann o Hermann Hesse (Gilgamesh sembra rappresentare il prototipo del «Suchende»). Ma sarebbe davvero impossibile anche solo citare tutti gli autori moderni che sono stati influenzati dal poema, o che vi fanno riferimento nelle loro opere. Rimandiamo quindi ad alcuni lavori recenti che possono fornire un'informazione ricca e variegata della 'fortuna' del poema: in una recente raccolta di studi (Gilgamesh, a Reader: v. Maier 1997), una parte consistente è dedicata a Influences of Gilgamesh on Later Literature e comprende studi quali Gilgamesh in the Arabian Nights (S. Dalley) o Gilgamesh and Philip Roth's Gil Gamesh (M. Colakis). Un altro volume interessante al riguardo è Gilgamesh Among Us: Modern Encounters with the Ancient Epic (v. Ziolkowski 2011). Gli Atti di un convegno dedicato ai parallelismi tra il poema di Gilgamesh e altre tradizioni letterarie sia antiche che modern ${ }^{38}$ sono stati pubblicati recentemente in un volume della rivista KASKAL (2012): tra i contributi, ricordiamo quelli di Peter De Ville (Gilgamesh: Some Suggestions for Literary Interpretation) e di A.R. George (The Mayfly on the River: Individual and Collective Destiny in the Epic of Gilgamesh).

Passando dalla letteratura ad altri ambiti di studi o altri generi di intrattenimento, si può dire che il richiamo di Gilgamesh e la sua universalità non hanno limiti. Ci limitiamo a citare brevemente solo alcuni esempi significativi: in un volume del 1992 (Forests: The Shadow of Civilization), Robert Pogue Harrison cita anche Gilgamesh e l'episodio nella Foresta dei Cedri; il poema, o alcuni suoi brani, sono stati musicati da diversi autori; infine, sono ormai numerose le serie a fumetti dedicate a Gilgamesh, che viene citato, come esempio di eroe universale e interplanetario, anche in un episodio della serie televisiva Star Trek, The Next Generation.

37 Moran 1980.

38 'Decoding Gilgamesh'. Conference Held on 12th-14th April 2010 at St John's College, Cambridge. 
La storia di Gilgamesh ha anche dato luogo a svariate interpretazioni e proposte di collegamento con altri cicli mitologici, di cui non possiamo dare conto in questa breve sintesi. ${ }^{39} \mathrm{Ci}$ sembra tuttavia significativo, a conclusione di questa parte generale di presentazione della saga, riportare un commento di Giorgio Buccellati, che ne offre un'interessante lettura in chiave sapienziale: ${ }^{40}$

La ricerca della vita non deve essere più considerata, se la mia lettura è corretta, come il tema centrale del poema. Certo, Gilgamesh è pur sempre presentato come l'eroe che va in cerca di fama e poi, dopo l'esperienza dell'amicizia e della morte di Enkidu, in cerca della vita: ma ciò diventa, in effetti, un pretesto narrativo per mostrare ben altra tesi. L'enfasi è spostata dall'oggetto della ricerca, la vita, allo sforzo stesso della ricerca in quanto tale, ai presupposti su cui è basata e alle conseguenze cui conduce: queste conseguenze non sono esterne, come lo sarebbe il perseguimento di un bene, foss'anche la vita fisica, ma invece sono interne, profondamente psicologiche e si accentrano sul mutamento spirituale del soggetto che la ricerca ha intrapreso. Perciò la conclusione è compiuta e perfetta con la tavoletta XI: Gilgamesh non è un campione temporaneamente sconfitto e a cui resta solo da ritentare, ma invece un uomo per cui la sconfitta diventa il punto d'inizio per una nuova comprensione delle vere dimensioni umane della vita. È una conclusione malinconica e inconcludente da un punto di vista eroico; da un punto di vista sapienziale, invece, è una conclusione piena e che non ammette ulteriori sviluppi.

\section{LE TRADIZIONI DI GILGAMESH NELL'ANATOLIA ITTITA}

Nel contesto dell'ampia diffusione, tra i secoli XV e XIII a.C., delle tradizioni relative all'eroe Gilgamesh in molte parti del Vicino Oriente antico (v. sopra $\mathbb{S}$ con relativa bibliografia), il caso dell'Anatolia ittita si contraddistingue in maniera notevole per la sua peculiarità. Dagli archivi della capitale ittita Hattusa ci sono infatti

39 Si veda in proposito il capitolo relativo alle Interpretazioni dell'epopea in Pettinato 1992, 38 ss.

40 Buccellati 1972, 34; il commento di Buccellati è riportato anche da Pettinato 1992, 41. Sulla lettura della versione canonica del poema di Gilgamesh in chiave sapienziale insistono anche George 2003 (per es. a p. 33), van der Toorn 2007 e Sallaberger 2013,73 ss. 
pervenute differenti versioni del poema di Gilgamesh, databili ad epoche diverse, in tre lingue differenti: accadico, hurrita e ittita. Il fenomeno è riconducibile alle modalità di trasmissione delle tradizioni mesopotamiche in ambito ittita, che giunsero in Anatolia attorno alla metà del XV secolo, soprattutto tramite il filtro culturale della regione di Kizzuwatna. ${ }^{41}$ Le tradizioni fiorite in ambito ittita sull'eroe Gilgamesh e le differenti versioni che ce le hanno conservate sono state oggetto di numerosi ed approfonditi studi, ai quali rimandiamo, limitandoci qui di seguito ad alcune osservazioni generali, funzionali alla presentazione che faremo in seguito dei due nuovi frammenti. ${ }^{42}$ Una premessa è tuttavia necessaria: lo stato frammentario della documentazione, soprattutto di quella in lingua accadica e hurrita - solo per le versioni in lingua ittita si può giungere a ricostruire almeno parzialmente un testo continuo -, lascia purtroppo senza risposta alcune fondamentali questioni relative alla genesi delle differenti redazioni e ai loro rapporti reciproci.

Esaminiamo qui di seguto le versioni ritrovate a Hattusa nelle tre diverse lingue (accadico, hurrita e ittita). La suddivisione dei tre gruppi testuali segue l'ordinamento dato nel Catalogue des textes hittites di E. Laroche $^{43}-$ (d'ora innanzi abbreviato come CTH) - e ripreso nella Konkordanz online dei testi ittiti presente sul sito web HPM Hethitologie Portal Mainz.

41 Questo è il nome di una regione situata nel sud-est dell'Anatolia, al confine con la Siria, grosso modo identificabile con la Cilicia di età classica, in cui convivevano tradizioni culturali hurrite, luvie e nord-semitiche e che giocò un ruolo fondamentale nella trasmissione di elementi culturali hurriti e mesopotamici agli Ittiti. Centro principale era la città di Kummanni, che secondo recenti ipotesi può forse essere identificata con il sito moderno di Sirkeli. Il territorio di Kizzuwatna fu annesso allo stato ittita in maniera definitiva nella seconda metà del XV secolo a.C.

42 Tra gli studi generali più recenti sulla tradizione relativa a Gilgamesh in ambito ittita rimandiamo in particolare a: Beckman 2003; Klinger 2005, 113 ss.; Haas 2006, 272 ss.; Archi 2007, 186 ss.; Bachvarova 2016, 54 ss. Sempre utile è l'ottima trattazione da parte di del Monte 1992, 285 ss. e 382 ss., che fornisce una traduzione italiana delle versioni in ittita e in accadico. Per una traduzione in inglese delle versioni in lingua ittita v. Beckman 2001, 157 ss.

43 E. Laroche, Catalogue des textes hittites, Paris 1971, con successivi supplementi. 


\subsection{I testi in lingua accadica da Hattusa}

I frammenti in lingua accadica (CTH 341.I ${ }^{44}$ permettono di ricostruire tre differenti redazioni: un gruppo di frammenti, databili in base al tipo di scrittura tipicamente medio-ittita al XV-XIV secolo e probabilmente appartenenti alla medesima tavoletta, presenta analogie, anche dal punto di vista linguistico, con la versione paleobabilonese del poema di Gilgamesh dal titolo «Colui che eccelle sopra i re» (v. sopra $\$ 2$ ), da cui i frammenti di Hattusa sembrano derivare direttamente. Essi contengono $i$ seguenti episodi: l'addomesticamento di Enkidu, la spedizione alla Foresta dei Cedri, il sogno premonitore di morte da parte di Enkidu. Un frammento minore $\left(\mathrm{KUB}^{45} 37.128\right)$ appartiene forse ad una tavoletta di importazione esterna, mentre il frammento KUB 4.12, databile paleograficamente alla fine del XIII secolo, rappresenta una rielaborazione locale di ambito ittita, contenente i sogni di Gilgamesh prima dello scontro con Huwawa - forma più antica del nome Humbaba, v. più avanti n. 50 - e l'episodio del Toro Celeste, precedentemente noto solo da un poemetto sumerico e assente invece apparentemente nelle versioni paleobabilonesi. ${ }^{46}$

\subsection{I testi in lingua hurrita da Hattusa}

Il contenuto dei frammenti in lingua hurrita (CTH 341.II) $)^{47}$ è difficilmente ricostruibile a causa del loro stato assai frammentario e dei problemi che ancora sussistono nella nostra conoscenza del lessico di questa lingua. Ciò che sembra ricostruibile con certezza è l'esisten$\mathrm{za}$, anche in questo caso, di almeno tre versioni differenti. Una prima versione (Salvini - Wegner 2004 Nr. 1), databile in base al tipo di scrittura al XIV secolo, reca nel colophon ${ }^{48}$ l'indicazione $\check{S} A$ D bíl-ga-

44 Per le versioni in lingua accadica l'edizione di riferimento, con trascrizione e traduzione, è ora George 2003, 306 ss. Uno studio accurato di questi frammenti si trova anche in Anthonioz 2012, 141 ss.

45 Abbreviazione di Keilschrifturkunden aus Boghazköi, serie in cui sono stati editi volumi contenenti copie autografiche di tavolette ittite.

46 Un riferimento al Toro Celeste compare anche nella supposta III tavola della versione ittita (v. più avanti).

47 I frammenti in lingua hurrita sono editi da Salvini - Wegner 2004 (testi Nr. $1-3,4$ ?, 5$)$, ai quali va aggiunto il frammento edito da Nakamura 2007.

48 Con questo termine si intende la parte finale di una tavoletta cuneiforme, in 
$m i \tilde{s}^{49}$ «di Gilgamesh», mentre una seconda versione (Salvini - Wegner 2004 Nr. 2), databile paleograficamente al XIII secolo, è indicata nel colophon come $\check{S} A$ Hu-wa-wa «di Huwawa»; $;^{50}$ in entrambe queste

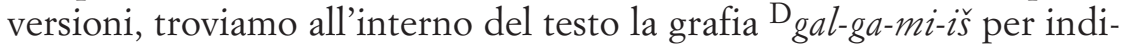
care il nome di Gilgamesh, che dunque compare, come anche nelle versioni ittite, divinizzato per mezzo del determinativo usato per indicare i teonimi ( $\mathrm{D}=$ sum. $\mathrm{DINGIR}$ «dio»). L'esistenza di una terza versione, databile paleograficamente al XIII secolo, è testimoniata dal frammento Salvini - Wegner 2004 Nr. 3, in cui il nome dell'eroe compare nella grafia ${ }^{D}$ GIŠ.GIM.MAŠ, che rappresenta la forma corrente di epoca mediobabilonese e che si ritrova anche nelle versioni in lingua ittita.

La tradizione hurrita si caratterizza anche per la presenza di elementi originali hurriti, tra i quali, per esempio, la menzione del dio della Tempesta hurrita Teššup di Kumme ${ }^{51} \mathrm{o}$ ancora il nome della taverniera, chiamata Nahmazuli, ${ }^{52}$ e quello dell'eroe del Diluvio, chiamato Ullu, che pure si ritrova nelle versioni ittite, invece di Uta-napishtim. ${ }^{53}$ Questo indica da un lato che i frammenti in lingua hurrita ci conservano non tanto una traduzione diretta di originali sumerici e/o babilonesi, quanto piuttosto una rielaborazione con adattamenti creati in ambiente hurrita delle tradizioni legate a Gilgamesh; dall'altro, come si preciserà

cui sono contenute alcune indicazioni tecniche volte a descrivere il testo in essa contenuto, quali per es.: il numero di tavoletta all'interno di una serie di più tavolette; il tipo di composizione; un eventuale 'titolo' della composizione; il nome dello scriba che ha redatto la tavoletta, ecc. Per una raccolta completa dei colophoni delle tavolette ittite v. ora Waal 2015.

49 Sulle diverse forme del nome di Gilgamesh nei testi di provenienza anatolica v. in particolare Beckman 2003, 50 e 57; George 2003, 79 ss. Più in generale, sul problema dell'origine e della spiegazione, peraltro ancora dibattuta, del nome Gilgamesh, v. i recenti contributi di Rubio 2012, Nurullin 2012 e Keetman 2014.

50 Huwawa è la forma più antica del nome del guardiano della Foresta dei Cedri, che risale all'epoca paleobabilonese e che troviamo anche nella versione ittita; nella versione canonica babilonese, come si è visto sopra, la forma del nome è invece Humbaba.

51 Nelle tradizioni sumerica e babilonese il dio della Tempesta (sum. Iškur, accad. Adad) non compare tra i personaggi protagonisti dell'epopea di Gilgamesh.

52 Su questo nome v. Haas 2003. Lo stesso nome, nella variante Nabmizuli, compare anche nella versione in lingua ittita; v. $\$ 6.3$.

53 Sull'interpretazione di questo nome come nominativo del pronome accadico ullû «quello, il distante», v. Beckman 2003, 52. 
ulteriormente in seguito, che la tradizione hurrita su Gilgamesh influenzerà, almeno in parte, le versioni del poema in lingua ittita. Va inoltre sottolineato che la nostra conoscenza dei testi in lingua hurrita relativi a Gilgamesh si deve all'opera degli scribi ittiti operanti presso la corte di Hattusa, che recepirono e fissarono per iscritto tale tradizione.

Non è possibile stabilire con certezza dove e quando si sia formata la tradizione hurrita relativa all'eroe Gilgamesh. L'esistenza di una serie evidentemente incentrata sulla figura di Huwawa è riconducibile al particolare interesse che questo episodio, tra le gesta di Gilgamesh, incontrò in epoca paleobabilonese (v. Fleming - Milstein 2010, 7 s.). A una relativa antichità della tradizione hurrita su Gilgamesh e all'ambiente nord-siriano potrebbe rimandare l'esistenza della già menzionata placchetta risalente alla fine del III millennio ritrovata a Urkesh, in cui sarebbero raffigurati, secondo l'interpretazione di Kelly Buccellati 2006, Gilgamesh ed Enkidu (v. sopra \$4).

\subsection{I testi in lingua ittita da Hattusa}

Per quanto concerne $\mathrm{i}$ testi in lingua ittita (CTH 341.III) ${ }^{54}$ essi sono in numero molto maggiore rispetto a quelli in accadico e in hurrita ritrovati a Hattusa e, pur nella loro frammentarietà, permettono una ricostruzione almeno parziale di un testo continuo: dall'insieme dei vari esemplari si può ricavare infatti l'esistenza di un racconto unitario che doveva coprire tre tavole (il testo indicato come CTH 341.III.1-3 nell'edizione di Rieken et al. del 2009), anche se, come vedremo, questa numerazione non è priva di incertezze. L'estensione del poema in lingua ittita era dunque sensibilmente inferiore rispetto a quella della versione canonica del poema babilonese in dodici tavole.

Anche nel caso dei testi in lingua ittita possiamo tuttavia riconoscere l'esistenza di diverse redazioni e/o versioni, che si distinguono tra loro per varianti più o meno marcate. ${ }^{55}$ In genere si tratta di esemplari che possono essere classificati o come duplicati, cioè contenenti un

54 L'edizione di riferimento dei frammenti che compongono le versioni in lingua ittita, in trascrizione e traduzione, è ora quella a cura di Rieken et al. 2009, disponibile online sul sito HPM - Hethitologie Portal Mainz all'indirizzo bttp://www.hethport.uni-wuerzburg.de/txhet_myth/textindex.php? $g=m y t h \varepsilon x=x$.

55 Cfr. a tal proposito quanto di recente osservato in Bachvarova 2016, pp. 63 ss. con rimandi a bibliografia precedente. 
testo praticamente identico, salvo minime varianti grafiche, oppure come redazioni parallele, contenti quindi parti di testo talora sensibilmente divergenti tra loro, ma di argomento affine. La distribuzione del testo sui diversi manoscritti spesso non coincide, così come la dimensione delle rispettive colonne su cui il testo è contenuto. Da tutti questi elementi emerge dunque che non esisteva un'unica versione canonica del poema ittita. ${ }^{56}$

Nonostante la necessaria cautela dovuta alle numerose lacune e varianti testuali, si può ricostruire nel modo seguente il contenuto del testo ittita del poema:

- Ia tavola (CTH 341.III.1): l'unico colophon superstite di questa tavola non contiene la numerazione, andata perduta in lacuna. ${ }^{57}$ Che si tratti di una «prima» tavola è tuttavia assicurato dal fatto che essa contiene il proemio del testo. La narrazione comincia con la descrizione della creazione di Gilgamesh da parte degli dèi e delle sue qualità fisiche; prosegue poi con l'arrivo di Gilgamesh a Uruk, la creazione di Enkidu, il suo incontro con Gilgamesh e la spedizione dei due amici alla Foresta dei Cedri, per terminare con il racconto della lotta di Gilgamesh ed Enkidu contro Huwawa. Di fatto, è questa la tavola meglio conservata, ricostruible sulla base di almeno nove esemplari, dalla quale è possibile ricavare una narrazione continua e confrontabile con la versione canonica babilonese del poema. Da ciò risulta che il contenuto di questa prima tavola corrispondeva a quello delle prime cinque tavole della verisone canonica. Sul testo della prima tavola torneremo poi nello specifico, poiché i due nuovi frammenti che presentiamo sono ulteriori manoscritti che appartengono proprio a questa tavola (v. più avanti $\$ 7.1-2$ ).

- II tavola? (CTH 341.III.2): non vi sono colophoni conservati, per

56 Va inoltre aggiunto che, accanto a questi frammenti che permettono la ricostruzione di un testo continuo, anche se lacunoso e con numerose varianti, esiste poi un gruppo di frammenti la cui collocazione all'interno di un testo unitario risulta oltremodo problematica, se non impossibile. In alcuni di essi, tra l'altro, si trovano elementi estranei alla tradizione sumero-accadica, come per es. un episodio che vede giungere Gilgamesh fino al mare. Essi sono catalogati nell'edizione di Rieken et al. 2009 come CTH 341.III.4-19; v. anche del Monte 1992, 294 e 392 s..

57 Esso si trova sul manoscritto CTH 341.III.1.E e ci tramanda il nome dello scriba che lo ha redatto: «Ta[vola ...], mano di Ipizzi»; su Ipizzi e la sua origine hurrita v. Klinger 2005, 121; de Martino 2011, 56. 
cui la numerazione è ipotetica e basata sostanzialmente sul fatto che il contenuto di questi frammenti risulta essere la continuazione di quello della prima tavola. Si tratta della tavola più lacunosa, di cui ci restano solo poco più di 30 righe di testo. Nella parte iniziale sembra essere narrata la morte di Huwawa ${ }^{58}$ poi vi sono il ritorno a Uruk dei due amici e un dialogo tra Gilgamesh e la dea Ishtar.

- III tavola (CTH 341.III.3): la numerazione di questa tavola è problematica. Se dal punto di vista narrativo i frammenti che la compongono, e che appartengono ad almeno cinque manoscritti diversi, rappresentano una prosecuzione rispetto alle due precedenti tavole, il colophon conservato in uno degli esemplari contiene l'indicazione «Prima tavola». ${ }^{59}$ L'incongruenza purtroppo non trova una spiegazione certa; si può forse ipotizzare un errore da parte dello scriba estensore della tavoletta. Per quanto concerne i contenuti, in questa tavola sono narrati: il sogno premonitore di morte da parte di Enkidu, le peregrinazioni di Gilgamesh dopo la morte dell'amico, l'incontro di Gilgamesh con la taverniera Ziduri/Nahmizuli, l'incontro di Gilgamesh con il traghettatore Urshanabi e infine l'incontro del nostrio eroe con un personaggio di nome Ullu, nel quale va identificato Uta-napishtim della tradizione babilonese (v. sopra $\$$ 6.2). Questa sezione del racconto corrisponde dunque alle tavole VII-X della versione canonica.

Come si evince da questa sommaria descrizione, l'estensione del poema in lingua ittita risulta decisamente inferiore rispetto a quella della versione canonica ${ }^{60}$ e, seppur più breve, sembra corrispondere nelle sue

58 Questa parte del testo CTH 341.III.2.A, corrispondente al frammento KUB 8.54 I 1-3, è omessa nell'edizione di Rieken et al. 2009; per questo passo e la sua collocazione v. del Monte 1992, 289.

59 Si tratta dell'esemplare CTH 341.III.3.A. Il testo del colophon recita: «Prima tavola del Canto di Gilgamesh». Sulla definizione di «canto» v. più avanti $\$ 7.1$.

60 La prima tavola corrisponde alle tavole I-V della versione canonica, mentre la supposta terza tavola corrisponde alle tavole VII-X. Sulla scorta di questo calcolo, risulta problematica l'estensione della supposta seconda tavola ittita, in quanto il suo contenuto corrisponderebbe a quello della sola VI tavola della versione canonica, con un rapporto pertanto assai diverso con questa versione rispetto a quanto avviene con le altre tavole. Data la lacunosità del testo ittita è tuttavia difficile giungere ad una ricostruzione sicura della sua suddivisione in diverse tavole e quella qui proposta, ripresa dall'edizione di Rieken et al. 2009, ha dunque il solo scopo di raggruppare ed ordinare un cospicuo numero di frammenti che forniscono una narrazione unitaria. 
linee essenziali a quella delle versioni di epoca paleobabilonese, iniziando con la creazione di Gilgamesh e terminando con il suo incontro con Utanapishtim. Maggior spazio è senza dubbio riservato alla narrazione della spedizione di Gilgamesh ed Enkidu alla Foresta dei Cedri: questo può essere dovuto sia all'influenza della tradizione paleobabilonese, che insiste in modo particolare su questo tema, sia anche ad un peculiare interesse che questo episodio doveva suscitare in ambito anatolico, in virtù della localizzazione nord-occidentale della Foresta dei Cedri, situabile tra le montagne del Tauro o dell'Amano (v. più avanti n. 79). ${ }^{61}$

Accanto alle evidenti analogie con il racconto di tradizione babilonese, il testo ittita presenta tuttavia alcune evidenti discrepanze. Queste differenze rispetto alle versioni babilonesi, di epoca sia antica che recente, sono state esaminate in maniera dettagliata da G. Beckman nel suo studio del 2003 (Beckman 2003) e riprese poi da A. Archi (Archi 2007). Ad essi rimandiamo per una panoramica dettagliata, limitandoci a osservare come tali differenze riguardino tanto aspetti di tipo strutturale e contenutistico - per es. il fatto che Gilgamesh giunga a Uruk, laddove nella tradizione sumerica e babilonese Uruk è la sua patria, oppure il fatto che la Foresta dei Cedri ${ }^{62}$ sia da localizzare nelle aree montagnose del Tauro o dell'Amano invece che nel Libano -, quanto aspetti di tipo formale e linguistico, con la presenza di stilemi attestati in altri testi letterari ittiti di origine hurrita (i cosiddetti «canti»; v. più avanti \$ 7.1) e con la presenza di nomi e/o personaggi estranei alla tradizone mesopotamica, ed ereditati piuttosto dalla tradizione hurrita. È il caso, per es., del nome di Uta-napishtim, che nella versione in lingua ittita è il medesimo di quella hurrita, Ullu, o ancora della presenza, in un frammento ittita, del nome di Impaluri, che sappiamo essere in hurrita il vizir del dio del Mare, personaggio non presente nell'epica babilonese di Gilgamesh.

Da ciò emerge come anche la tradizione ittita su Gilgamesh non possa venir considerata come una semplice e diretta traduzione di un originale babilonese, ma sia frutto di un complesso processo di ricezione e adattamento di tradizioni sia sumeriche e babilonesi, sia hurrite. I

61 Per una valutazione generale delle differenze tra la versione ittita e quelle babilonesi v. Tigay 1982, 118.

62 Nella versione ittita la Foresta dei Cedri viene indicata semplicemente come "montagne" per mezzo dell'ideogramma sumerico HUR.SAGMEŠ. 
frammenti ittiti sono tutti databili al XIII secolo a.C., sia dal punto di vista paleografico che da quello linguistico, tanto che non sembra possibile porre la nascita della tradizione in lingua ittita su Gilgamesh ad un periodo precedente. È tuttavia difficile stabilire a cosa sia dovuta la commistione di diversi elementi (sumerico-babilonesi e hurriti) presenti nella tradizione ittita, se essa sia cioè il risultato di un'operazione di contaminazione da parte ittita di elementi di diversa provenienza, oppure se gli scribi ittiti abbiano recepito un modello hurrita già contaminato e lo abbiano semplicemente tradotto nella loro lingua. A nostro avviso la prima ipotesi è la più probabile: $i$ testi in lingua ittita relativi alle gesta dell'eroe Gilgamesh sono il prodotto di una raffinata operazione culturale e letteraria da parte degli scribi ittiti, che hanno recepito e rielaborato, adattandole al proprio orizzonte e patrimonio culturale, tradizioni di diversa origine, sia sumerico-babilonese che hurrita. ${ }^{63}$ Quella che viene spesso definita una semplice 'parafrasi' o 'traduzione' ittita del testo di Gilgamesh sembra mostrare piuttosto le caratteristiche di un'opera complessa ed originale.

\section{I DUE NUOVI FRAMMENTI (Figg. 4-6)}

La parte finale del nostro contributo è dedicata alla presentazione dei due nuovi frammenti, d'ora innanzi etichettati come ' $\mathrm{P}$ (rivato) 1 ' e 'P(rivato) 2', che hanno fornito l'occasione di ripercorrere nelle sezioni precedenti l'affascinante e complessa storia delle tradizioni legate alla leggendaria figura dell'eroe Gilgamesh. Poiché sussitono ancora alcuni problemi esegetici nell'esatta ricostruzione del testo dei due frammenti, si è scelto di darne qui di seguito una descrizione generale, evidenziando in particolar modo quei passi in cui i due frammenti apportano delle novità rispetto al testo già noto; si intende invece riservare ad altra sede una trattazione tecnica più approfondita, con traslitterazione completa e commento filologico, in cui si discutono i problemi interpretativi ancora presenti soprattutto nel frammento P 2, che risulta di lettura particolarmente difficile a causa del cattivo stato di conservazione.

63 Siamo di contro poco propensi ad accettare la recente ipotesi formulata da Bachvarova 2016, che vede nel testo ittita di Gilgamesh il prodotto di una tradizione orale. 
I due frammenti, per dimensioni e comuni caratteristiche paleografiche ${ }^{64}$ dovrebbero appartenere alla medesima tavoletta, pur non congiungendosi direttamente tra loro e mostrando una colorazione in parte differente. ${ }^{65} \mathrm{Il}$ frammento $\mathrm{P} 1$, inscritto solo sulla faccia anteriore (recto) - quella posteriore (verso) non presenta tracce di scrittura -, rappresenta la parte iniziale sinistra della prima colonna, mentre il frammento P 2, inscritto su entrambe le facce, contiene sul recto la parte finale della seconda colonna e sul verso la parte iniziale della terza. Il verso anepigrafo di P 1 costituisce invece la parte finale sinistra della quarta colonna.

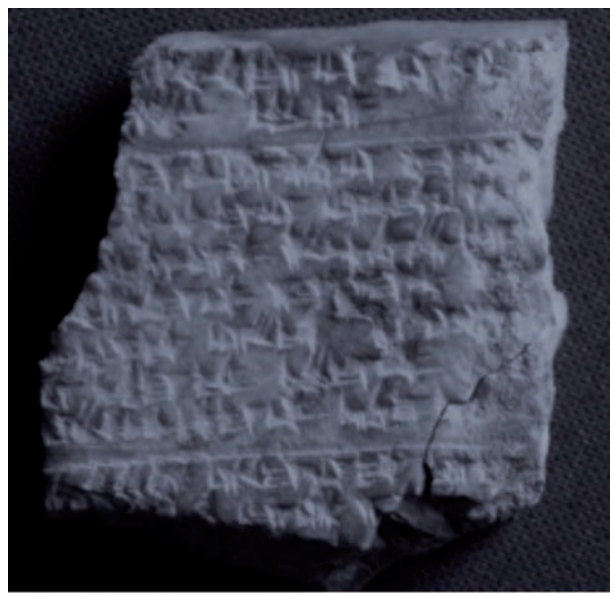

Fig. $4-P$ (rivato) 1 recto

64 Si tratta chiaramente, dal punto di vista della forma dei segni cuneiformi, di manoscritti della seconda metà del XIII sec. a.C. È particolarmente indicativa in questo senso la forma del segno LI nel frammento $\mathrm{P} 1 \mathrm{r}$. 5 . Anche alcune peculiarità linguistiche portano a datare la redazione dei frammenti all'epoca del XIII sec.

65 Le misure dei due frammenti sono le seguenti: P 1 larghezza $5,2 \mathrm{~cm}$., altezza $6 \mathrm{~cm}$., spessore massimo $2 \mathrm{~cm}$.; P 2 larghezza $9,3 \mathrm{~cm}$., altezza 4,7 cm., spessore massimo $2,2 \mathrm{~cm}$.. Seguendo l'ipotesi che i due frammenti appartengano alla medesima tavoletta la larghezza di P 1 dovrebbe corrispondere a circa metà della larghezza originaria di una colonna di testo, mentre la larghezza del frammento P 2 dovrebbe corrispondere all'incirca alla larghezza di un'intera colonna di testo. Secondo questa ipotesi ricostruttiva, la tavoletta doveva dunque misurare circa $20 \mathrm{~cm}$. in larghezza. Non è invece possibile calcolare l'altezza complessiva della tavoletta sulla base dei due frammenti. 


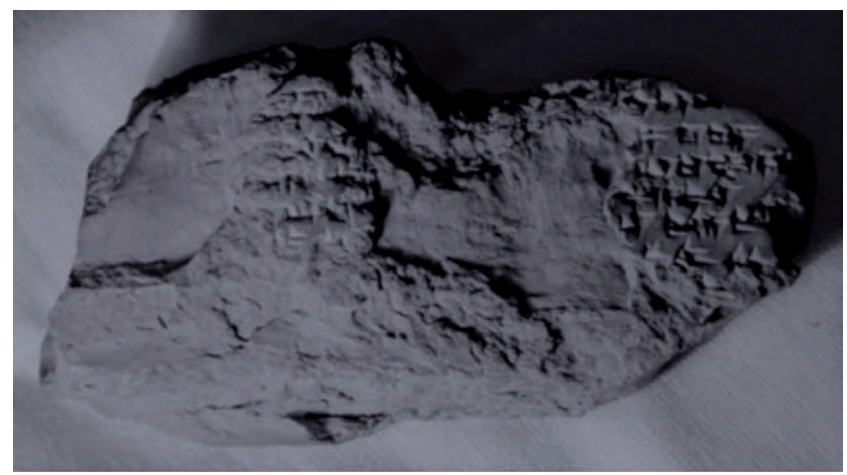

Fig. 5 - $P$ (rivato) 2 recto

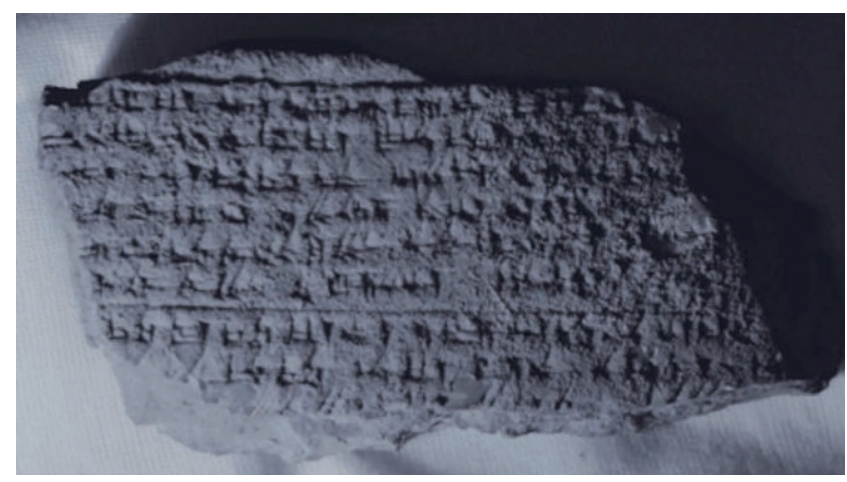

Fig. 6 - P(rivato $) 2$ verso

\subsection{Il frammento $P$ (rivato) 1}

Il frammento P 1 (v. Fig. 4) risulta essere nel recto un duplicato del frammento KUB 8.57+, che appartiene all'esemplare CTH 341.III.1.A+H secondo la ricostruzione di Rieken et al. 2009, e che contiene la parte iniziale, con l'incipit, della prima tavola della versione ittita in tre tavole descritta sopra al $\$ 6.3$. La corrispondenza tra i due frammenti è strettissima: le undici righe di testo parzialmente conservate sul recto di P 1 coincidono con le rr. 1-11 del recto di KUB 8.57+; analoga è pure la suddivisione del testo in paragrafi nei due esemplari. Inoltre, anche il frammento KUB 8.57+, come P 1, ha il verso anepi- 
grafo. Ciò porta a ritenere che la tavoletta cui appartenevano i nostri due frammenti aveva probabilmente le medesime dimensioni della tavoletta CTH 341.III.1.A+H.

Nelle prime due righe di entrambi i manoscritti (P 1 I 1-2 = KUB $8.57+$ I 1-2) è conservato il proemio del poema ( $\$ 1$ della I tavola nell'edizione di Rieken et al. 2009), che si può ora ricostruire nella maniera seguente grazie all'apporto del frammento P 1: «[Ce]lebrerò lui, Gilgamesh [...], l'eroe, il re!». ${ }^{66}$ L'incipit della versione ittita, introdotto da un verbo alla prima persona singolare, è diverso sia da quello della versione canonica («Colui che vide la profondità, il fondamento del paese») ${ }^{67}$ sia da quello della principale versione paleobabilonese («Colui che eccelle sopra i re») $)^{68}$ e richiama l'espressione formulare tipica con cui venivano introdotti i poemi narrativi di origine hurrita, che nei colophoni venivano etichettati dagli scribi ittiti per mezzo dell'ideogramma sumerico Š̀̀R «canto». ${ }^{69} \mathrm{Ne}$ abbiamo alcuni esempi sia in lingua hurrita, dove veniva usato il verbo šir- (nella forma al volitivo «io voglio cantare/celebrare»), sia in lingua ittita, dove il verbo solitamente utilizzato era išhamai- «cantare» (nella forma di presente $1^{\mathrm{a}}$ pers. sing. išbamibhi); nel nostro testo viene invece utilizzato il verbo walla- «celebrare, lodare», che è caratteristico degli inni alle divinità. Queste formule introduttive dei poemi narrativi hurro-ittiti alla prima persona singolare, comuni anche agli inni, sono state di recente analizzate da $\mathrm{Ch}$. Metcalf ${ }^{70}$ che parla di «'Let-me-sing' topos», osservando come esse trovino corrispondenza nella tradizione sumero-accadica e siano giunte agli Ittiti per mezzo dell'influenza culturale hurrita.

66 Il frammento P 1 fornisce la definitiva conferma alla lettura della forma verbale iniziale come wallabhi «io esalterò/celebrerò», che era già stata ipotizzata da E. Laroche nel suo fondamentale studio sul lessico della preghiera ittita (Laroche 1964-65, 28 n. 8). La forma si può ricostruire combinando la lezione del frammento KUB 8.57 I 1 [wa]-al-la -[ con quella di P1 r. 1 [wa-al-l]a-ab-bi-.

67 Questo incipit è già attestato in età mediobabilonese in un testo ritrovato a Ugarit (RS 94.2006); v. George 2007 e, sempre su questo testo, v. più avanti.

68 Questo verso corrisponde alla r. 29 della I tavola della versione babilonese canonica.

69 Ricordiamo che nel colophon della supposta III tavola della versione ittita del poema (v. sopra n. 59) si trova l'ideogramma ŚİR «canto», a dimostrazione dell'influsso che la tradizione letteraria hurrita esercitò sulla versione ittita del poema di Gilgamesh; cfr. anche Tigay 1982, $51 \mathrm{s.}$

70 V. Metcalf 2015, 100 ss. 
La sezione successiva, alle rr. 3-9, corrispondenti alle rr. 3-9 del duplicato KUB 8.57+ ( $\$ 2$ della I tavola nell'edizione di Rieken et al. 2009), contiene la descrizione della creazione di Gilgamesh da parte degli dèi e delle sue qualità fisiche. Anche in questo caso il nuovo frammento aggiunge alcuni nuovi elementi di notevole interesse. In primo luogo, esso ci fornisce a r. 4 il nome delle divinità che «crearono» (itt. šamner) Gilgamesh, finora rimasto sconosciuto perché perduto in lacuna nel duplicato KUB 8.57+: si tratta delle dee del Fato (itt. ${ }^{\mathrm{D}} g u l-\check{s} u-$ $u s ̌) .{ }^{71}$ Dopo questo teonimo andrà integrato nella lacuna alla fine della

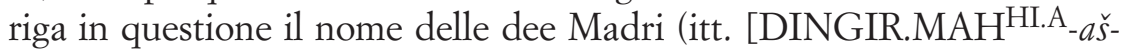
$\check{s}$ a]), normalmente associate alle dee del Fato all'atto della nascita dell'uomo. ${ }^{72}$ Resta invece purtroppo ignoto il nome della divinità che probabilmente «progettò»(?) Gilgamesh, che è perduto in lacuna in KUB $8.57+\mathrm{I} 2$ e non è conservato nemmeno nel nostro nuovo frammento.

Nelle righe successive si elencano le qualità che alcune divinità offrirono a Gilgamesh, contribuendo così alla sua creazione. Anche qui il frammento P1 fornisce un nuovo elemento: a r. 5 infatti è conservata la qualità che il dio Sole del Cielo fornisce a Gilgamesh, vocabolo invece andato perduto in KUB 8.57+. Si tratta dell' «animo da eroe» (itt. ba$a \grave{s}$-ta-li-ia-[tar). In questo modo, combinando i due duplicati, si possono ricostruire nel modo seguente le rr. 3-7:

(3) L'eroico dio ... [...-ò] (4) la persona/figura di Gilgameš, (come andava) creata; ma le dee del Fato [ e le dee Madri] la crearono, (5) la persona/figura ${ }^{73}$ di Gilgameš; a lui il dio Sole del Cielo diede un animo d'eroe; (6) il dio della Tempesta invece gli diede la forza: (7) i grandi dèi [lo] c[rearono?], Gilgamesh.

Nella I tavola della versione canonica del poema, Gilgamesh è

71 Sulla lettura di questo nome divino v. ora Yakubovich 2014 (pace Waal 2014).

72 Su queste divinità e il loro collegamento con la nascita dell'uomo v. soprattutto Beckman 1983, 242 ss. e, di recente, Archi 2014. In particolare, A. Archi (p. 10 s.) analizza l'uso del verbo itt. šamnai- « creare, plasmare» in associazione a questi due gruppi di divinità, dimostrando come alla base vi sia l'idea dell'uomo creato a partire dall'argilla, una concezione, questa, di origine sumero-accadica, che gli Ittiti ereditarono per il tramite hurrita.

73 Il termine utilizzato è il sumerogramma ALAM, corrispondente all'accadico șalmu. 
detto essere figlio del leggendario re di Uruk Lugalbanda e della dea Ninsun (I 35-36), per due terzi dio e per un terzo uomo (I 48). Anche qui c'è il motivo della creazione da parte degli dèi della «forma» (accad. șalmu) del suo corpo; gli dèi menzionati sono in questo caso Bēlet-ilī, la grande madre degli dèi, ed Enki/Ea, il demiurgo divino (menzionato con l'epiteto di Nudimmud), entrambi normalmente associati alla creazione dell'uomo nella tradizione mesopotamica (I 49-50). È interessante osservare la variazione che ritroviamo di questo motivo nella versione ittita: i genitori di Gilgamesh non sono mai menzionati, mentre le divinità che concorrono alla creazione di Gilgamesh sono da un lato quelle principali del pantheon ittita (dio Sole del Cielo e dio della Tempesta), dall'altro le divinità tradizionalmente associate alla nascita dell'uomo nella religione ittita, le dee del Fato e le dee Madri. Questo motivo, che rielabora tradizioni mesopotamiche sulla nascita dell'uomo, è giunto in ambito ittita, come è già stato osservato in precedenza, per la mediazione della cultura religiosa hurrita. ${ }^{74}$

Nelle righe successive del testo, che completano la celebrazione dell'eroe Gilgamesh dal punto di vista del suo aspetto fisico, sono descritte le dimensioni eccezionali del suo corpo (P 1 I 7-9 = KUB $8.57+$ I 7-9). In questo caso il nuovo frammento aggiunge poco, se non la conferma della lettura a r. 9 del termine UZUHAR-ni-uš-ma-aš-š $i$ indicante una parte del corpo dal significato purtroppo oscuro.

Questa parte del testo può essere dunque resa nel modo seguente:

La sua persona/figura (era) 11 cubiti (ca. $550 \mathrm{~cm}$.) (n) $^{75}$ in [tezza]; (8) il suo petto invece (era) 9 ....... in larghezza (9) e il suo barniu? 3 [...] in lunghezza.

Questa parte di testo con le indicazioni delle misure di Gilgamesh si ritrova anche nella I tavola della versione canonica, immediatamente dopo il riferimento alla creazione di Gilgamesh da parte degli dèi, proprio come nella versione ittita. Questa porzione di testo è conservata in

74 Si può tra l'altro osservare una certa somiglianza tra questa descrizione della 'creazione' di Gilgamesh, cui i grandi dèi contribuiscono, con quella della 'creazione' del dio della Tempesta hurrita Teššup in un testo mitologico di origine hurrita, il cosiddetto «Canto dell'uscire» (CTH 344).

75 L'unità di misura utilizzata nel testo ittita e qui resa con «cubito» è l'accadogramma AMMATU, che si ritene corrispondesse a ca. $50 \mathrm{~cm}$. 
maniera lacunosa nella versione canonica, ma è oggi ricostruibile con sicurezza grazie all'apporto di un frammento del poema ritrovato ad Assur e grazie all'apporto del già menzionato testo mediobabilonese da Ugarit RS 94.2006. Nella ricostruzione fornita da A. George (2007, 247 s.) essa suona (I tavola rr. 52-58):

[A giant(?)] in stature, eleven cubits [was his height,]

[four cubits was] the width of [his chest,]

a triple cubit his foot, half a rod his leg,

six cubits was the length of his stride,

$[x]$ cubits the whiskers(?) of his cheeks.

Come giustamente osservato dallo stesso George, la descrizione delle dimensioni di Gilgamesh nel testo accadico «tallies more closely with the Hittite Gilgameš than previously realized» (George 2007, 248).

Le ultime due righe del frammento P 1 (10-11) coincidono con le rr. 10-11 di KUB 8.57+ (inizio del $\$ 3$ della I tavola nell'edizione di Rieken et al. 2009) e menzionano l'arrivo di Gilgamesh a Uruk (P 1 I 11 [URU $u$ ]-ra-ga URU-ri a-a[ $r=\mathrm{KUB} 8.57+\mathrm{I} 11\left[\mathrm{URU}_{\mathcal{u}}\right.$ ]-ra-ga URU-ri aar-aš) dopo un lungo peregrinare per diversi paesi. Abbiamo dunque in questo caso una notevole variazione rispetto alla tradizione mesopotamica, in cui Gilgamesh è un re originario di questa città.

\subsection{Il frammento $P$ (rivato) 2}

Il secondo frammento (P 2) contiene, come già anticipato, la fine della seconda colonna e l'inizio della terza colonna della I tavola della versione ittita del poema (corrispondente ai $\$ \$ 15^{\prime \prime \prime}-17$ '”' nella ricostruzione del testo di Rieken et al. 2009; ma v. più avanti). Anch'esso risulta essere, in alcune sue parti, duplicato di due testimoni attribuiti alla I tavola, ma aggiunge nel verso una nuova porzione di testo finora sconosciuta. La lacunosità e le difficoltà di lettura di questa parte della tavoletta, che su questa faccia risulta essere fortemente danneggiata da incrostazioni calcaree, impediscono purtroppo di ricostruire completamente questa nuova sezione di testo; tuttavia gli elementi essenziali possono essere riconosciuti e rappresentano un'importante novità nella ricostruzione globale della versione ittita del poema.

Nelle poche e lacunose righe conservate sul recto (v. Fig. 5), il frammento P 2 presenta evidenti analogie con KUB 60.14+ Ro II 
(esemplare CTH 341.III.1.J), anche se una ricostruzione sicura delle corrispondenze tra i due esemplari non sempre risulta possibile a causa del loro stato frammentario. Questa parte di testo, che nell'edizione di Rieken et al. 2009 rappresenta il $\mathbb{\$} 15^{\prime \prime \prime}$ della I tavola, alla fine della II colonna, contiene l'episodio in cui Gilgamesh annuncia alle sue truppe (P 2 II 1' KARAŠ = KUB 60.14+ II 5'), radunate per una festa/un banchetto (KUB 60.14+ II 7' EZEN 4 ), la spedizione contro Huwawa. ${ }^{76}$ È in questo contesto che nella versione ittita fa la sua comparsa, per la prima volta, il nome del guardiano della Foresta dei Cedri. Questo episodio, sebbene in forma più ridotta, trova riscontro nell'annuncio che Gilgamesh ed Enkidu fanno della loro spedizione agli anziani (accad. šibūtu) di Uruk nella versione paleobabilonese conservata sulla cosiddetta Tavoletta di Yale (v. George 2003, 192 ss.). Nella versione canonica (II tavola) l'annuncio della spedizione viene fatto inizialmente da Gilgamesh ai «giovani uomini» (accad. ettūtu), che ricordano da vicino le «truppe» della versione ittita, e solo successivamente da Enkidu agli anziani (accad. šibūtu / rabûtu). In entrambe le versioni accadiche gli anziani mettono in guardia Gilgamesh dai pericoli che la spedizione comporta, esortandolo a tornare sano e salvo; questa parte non si trova nella versione ittita.

Nell'esemplare finora noto, KUB 60.14+, è conservata in maniera assai frammentaria solo la parte iniziale del discorso di Gilgamesh, dove si cita il nome di Huwawa (KUB 60.14+ II 10' ' bu-w]a-wa-in-wa x-allu «io voglio [...]-re [Hu]wawa», seguendo la lettura di Rieken et al. 2009). Qui termina la colonna II di questo frammento e con queste parole si interrompeva anche il discorso di Gilgamesh, la cui prosecuzione ora è testimoniata dal verso di $\mathrm{P} 2$. L'ultima riga conservata del recto di $\mathrm{P} 2$ aggiunge una piccola parte alla frase iniziale del discorso di Gilgamesh («e quello [...]», P 2 II 6'); su questa faccia della tavoletta dovevano trovarsi ancora almeno due righe di testo, andate perdute.

Il discorso di Gilgamesh continua comunque nelle prime due righe del verso della tavoletta P 2 (v. Fig. 6); questo è assicurato dalla presenza della forma pronominale ammuk "io/me/a me" in P 2 III 2. In contesto frammentario si trova il riferimento ad un «santuario» (P 2 III 1 Éka-ri-ma-a-ni) e al «giorno futuro» (III 2 I-NA EGIR.UD.[KA]M[...]). Con questa riga lacunosa termina il discorso di

76 Su questa forma del nome v. sopra n. 50. 
Gilgamesh, cui fa immediatamente seguito, alla riga successiva, l'entrata in scena del dio Sole, che dialoga con l'eroe: «Giù dal cielo di[ce] il Dio Sole» (III 3 [n]u-kán ne-pi-ša-az! DUTU-uš GAM me-mi-iš-k[e-ez$z i])$. Anche il discorso del dio Sole è conservato in maniera frammentaria, ma sembra che in qualche modo la divinità inciti Gilgamesh a compiere la spedizione contro Huwawa, il cui nome si trova alla r. $6\left({ }^{\mathrm{D}} \mathrm{b}\right] u-$ wa-wa-in). Si noti in particolare la forma di imperativo con cui si apre il discorso del dio Sole a r. 4 ([i]-it-wa-ra-an «[V]a'!»).

Le rr. 1-6 del verso di P 2 ci testimoniano dunque una parte del testo, contenente la prosecuzione del discorso di Gilgamesh relativo alla sua impresa nella Foresta dei Cedri e l'intervento diretto sulla scena del dio Sole, che finora era rimasta sconosciuta, fatta eccezione per poche tracce di segno illeggibili presenti sul verso dei frammenti KUB 60.14 + KUB 36.72 (CTH 341.III.1.J+F). Secondo la ricostruzione del testo di Rieken et al. 2009 questa sezione rappresenterebbe il $\$ 16$ '"' della I tavola; tuttavia, in considerazione del fatto che qui prosegue il discorso di Gilgamesh iniziato nella parte finale della II colonna, è possibile che all'inizio della III colonna della I tavola si trovi piuttosto la prosecuzione del $\$ 15^{\prime \prime \prime}$, che comincia alla fine della II colonna.

L'intervento in questo contesto del dio Sole, che nel corso della narrazione assisterà Gilgamesh ed Enkidu nella loro spedizione alla Foresta dei Cedri e nel loro scontro con Huwawa, merita un breve commento. Nella versione canonica del poema, la richiesta di assistenza e di protezione al dio Sole per i due eroi è fatta dalla madre di Gilgamesh, la dea Ninsun (III tavola). Nella versione paleobabilonese conservata nella cosiddetta Tavoletta di Yale (v. sopra) è invece Gilgamesh che prega direttamente il dio Sole per ottenere il suo favore (fine della V colonna). ${ }^{77} \mathrm{Nel}$ passo della versione ittita ora presentato, che segna come detto l'entrata in scena del dio Sole ${ }^{78}$ non sappiamo se Gilgamesh

77 Sul mutamento che il ruolo del dio Sole subisce rispetto alla tradizione sumerica nella versione paleobabilonese del poema e poi in quella canonica v. Tigay 1982,76 ss.

78 Nel testo ittita, una preghiera (itt. arkuwar) al dio Sole sarà rivolta da Gilgamesh al momento dello scontro con Huwawa, in seguito alla quale il dio Sole invierà contro Huwawa i venti che lo immobilizzeranno, provocandone la sconfitta (I tavola, $\$ \$ 24$ '”' -25 '”' edizione di Rieken et al. 2009). Nel $\$ 25$ '”' probabilmente si fa riferimento, in maniera purtroppo frammentaria, al dialogo tra Gilgamesh e il dio Sole conservato nella nostra tavoletta. 
rivolga esplicitamente una preghiera al dio Sole. In ogni caso è assente la mediazione della madre di Gilgamesh, che, come già detto, non compare del tutto nella versione ittita. In questo il testo ittita si avvicina dunque maggiormente alla versione paleobabilonese, dove abbiamo un contatto diretto tra Gilgamesh e il dio Sole.

Le ultime tre righe del frammento P 2 (III 7-10) sono un duplicato di KUB 36.72+ III 2'-4' (CTH 341.III.1.F) e contengono l'inizio del $\$ 17^{\prime \prime \prime \prime}$ dell'edizione di Rieken et al. 2009 (o piuttosto $\$ 16^{\prime \prime \prime \prime ' ?), ~ d o v e ~ s i ~}$ narra del viaggio di Gilgamesh ed Enkidu verso la Foresta dei Cedri. Anche in questo caso il frammento P 2 aggiunge alcuni nuovi elementi. In particolare menzioniamo la parte iniziale di r. 7, che permette di ricostruire il verbo della prima frase del paragrafo: «si abbracciarono (Gilgamesh ed Enkidu)» (itt. a-ap-pa-an-ta-ti), che in KUB 36.72+ III 2' è invece perduto in lacuna. Inoltre in P 2 III 10 è conservato il numerale «30», perduto invece in KUB 36.72+, nella locuzione «per 30 miglia», riferita al percorso compiuto nel viaggio dai due eroi. Qui il nostro nuovo frammento si interrompe.

Il testo della versione ittita prosegue poi in KUB 36.72+ con l'arrivo di Gilgamesh ed Enkidu al fiume Mala (che secondo l'opinione corrente rappresenta il nome ittita del medio-alto corso dell'Eufrate) dove compiono un sacrificio. Successivamente i due eroi giungono, dopo 16 giorni di viaggio, alle montagne dove si trova la Foresta dei Cedri, che, come abbiamo detto, nella versione ittita è da localizzarsi nelle aree montagnose del Tauro o dell'Amano invece che nel Libano. ${ }^{79}$

79 Nella versione canonica la Foresta dei Cedri è localizzata sul Monte Libano (šad̂̀ Labnānu; cfr. anche la forma «Montagna dei Cedri»), localizzazione, questa, che già si trova in un frammento di età paleobabilonese (v. George 2003, 225), mentre le tradizioni più antiche pongono questo luogo a est del paese di Sumer, dove sorgeva il sole (Tigay 1982, 78; George 1999, 149 e 2003, 485 n. 133, 496; Beckman 2003, 44 n. 57; Fleming - Milstein 2010, 9, 29 n. 23). La versione ittita, nel situare la scena presso l'Eufrate, mostra la volontà da parte del redattore di spostare l'azione del racconto in un'area geograficamente collegata al territorio ittita, probabilmente la zona montagnosa dell'Amano o del Tauro (Beckman 2001, 157 e 2003, 44; su questo motivo cfr. anche Klinger 2005, 117; Archi 2007, 187; Bachvarova 2016, 71 n. 75). D'altra parte è interessante osservare come in una versione paleobabilonese (Schøyen 2 ; v. George 2003, 224 ss.) la meta del viaggio verso la Foresta dei Cedri cui giungono Gilgamesh ed Enkidu è il «Paese di Ebla». Qui siamo dunque in presenza di una tradizione che colloca nel territorio di Ebla le montagne dove si trovavano i cedri, tradizione che troviamo già documentata in un testo sumerico, come osserva George 2003, 225 s. e in altri testi relativi 


\section{BIBLIOGRAFIA}

Al-Rawi - George 2014 - F. Al-Rawi - A. R. George, Back to the Cedar Forest: The beginning and end of Tablet $V$ of the Standard Babylonian Epic of Gilgameš, Journal of Cuneiform Studies 66 (2014), 69-90.

Anthonioz 2012 - S. Anthonioz, Miroir d'une transmission du Gilgameš akkadien en périphérie (première partie), in C. Roche-Hawley, R. Hawley (éds.), Scribes et érudits dans l'orbite de Babylone, Paris, De Boccard, 2012, 139-145.

Archi 2007 - A. Archi, Transmission of recitative literature by the Hittites, Altorientalische Forschungen 34 (2007), 185-203.

Archi 2014 - A. Archi, The Anatolian Fate-Goddesses and their different traditions, in E. Cancik-Kirschbaum, J. Klinger, G. G. W. Müller (eds.), Diversity and Standardization. Perspectives on Social and Political Norms in the Ancient Near East, Berlin, Akademie Verlag, 2014, 1-26.

Arnaud 1987 - D. Arnaud, Emar VI/4. Textes de la bibliothèque: transcriptions et traductions, Paris, Editions Recherche sur les Civilisations, 1987.

Arnaud 2007 - D. Arnaud, Corpus des textes de bibliothèque de Ras Shamra-Ougarit (1936-2000) en sumérien, babylonien et assyrien (Aula Orientalis Supplementa, 23), Sabadell, Ausa, 2007.

Bachvarova 2016 - M. Bachvarova, From Hittite to Homer: The Anatolian background of ancient Greek epic, Cambridge, Cambridge University Press, 2016.

Beckman 1983 - G. Beckman, Hittite birth rituals (Studien zu den Boğazköy-Texten, 29), Wiesbaden, Harrassowitz Verlag, 1983.

Beckman 2001 - G. Beckman, The Hittite Gilgamesh, in B.R. Foster (ed.), The Epic of Gilgamesh, New York, Norton, 2001, 157-65.

Beckman 2003 - G. Beckman, Gilgamesh in Hatti, in G. Beckman, R. Beal, G. McMahon (eds.), Hittite studies in Honor of Harry A. Hoffner, Jr. on the occasion of his 65th birthday, Winona Lake, Ind., Eisenbrauns, 2003, 37-57.

Bottéro 1992 - J. Bottéro, L'Épopée de Gilgameš, Paris, Gallimard, 1992.

Buccellati 1972 - G. Buccellati, Tre saggi sulla sapienza mesopotamica - I, Oriens Antiquus 11 (1972), 2-36.

Buccellati in stampa - G. Buccellati, The First Gilgamesh. Conjectures about the earliest Epic (articolo in stampa in una Festschrift).

Cooper 1981 - J.S. Cooper, Gilgamesh and Agga: A review article, Journal of Cuneiform Studies 33 (1981), 224-241.

D’Agostino 2017 - F. D’Agostino, Gilgameš. Il re, l'uomo, lo scriba, Roma, L'asino d'oro edizioni, 2017.

alle spedizioni dei sovrani di Akkad verso il nord-ovest (v. George 2003, 93 s.). Questo porta ad identificare il luogo in cui Gilgamesh ed Enkidu vanno alla ricerca dei cedri verosimilmente con la montagna dell'Amano, non con il Monte Libano (v. ulteriori attestazioni di questa identificazione sempre in George 2003, 94), fornendo pertanto un parallelo con la localizzazione della spedizione alla Foresta dei Cedri che si trova nel testo ittita. 
Dalley 1991 - S. Dalley, Myths from Mesopotamia: Creation, the Flood, Gilgamesh and others, Oxford, Oxford University Press, 1991 (trad. it.: Il retaggio della Mesopotamia, Milano, Adelphi, 2016).

de Martino 2011 - S. de Martino, Hurrian personal names in the kingdom of Hatti (Eothen, 18), Firenze, LoGisma Editore, 2011.

del Monte 1992 - G.F. del Monte, Versione ittita, in Pettinato 1992, 285-299 e 382-397.

Falkenstein 1968 - A. Falkenstein, Gilgameš A. Nach sumerischen Texten, Reallexikon der Assyriologie III, 1968, 357-363.

Fleming - Milstein 2010 - D.E. Fleming - S.J. Milstein, The buried foundation of the Gilgamesh Epic: The Akkadian Huwawa narrative (Cuneiform Monographs, 39), Leiden - Boston, Brill, 2010.

Frayne 2010 - D. Frayne, Gilgameš in Old Akkadian Glyptic, in Steymans Hrsg. 2010, 165-208.

Gadotti 2014 - A. Gadotti, 'Gilgamesh, Enkidu, and the Netherworld' and the Sumerian Gilgamesh Cycle (Untersuchungen zur Assyriologie und Vorderasiatischen Archäologie, 10), Boston - Berlin, De Gruyter, 2014.

George 1999 - A. George, The Epic of Gilgamesh. A new translation, London, Penguin Books, 1999.

George 2003 - A. George, The Babylonian Gilgamesh Epic. Introduction, critical edition and cuneiform texts (2 voll.), Oxford, Oxford University Press, 2003.

George 2007 - A. George, The Gilgameš Epic at Ugarit, Aula Orientalis 25 (2007), 237 254.

Glassner 2004 - J.-J. Glassner, Mesopotamian Chronicles, Atlanta, Society of Biblical Literature, 2004.

Goetze - Levy 1959 - A. Goetze - S. Levy, A fragment of the Gilgamesh Epic from Megiddo, Atiqot 2 (1959), 121-128.

Gurney et al. 1957 - O.R. Gurney - J.J. Finkelstein - P. Hulin, The Sultantepe Tablets, London, The British Institute of Archaelogy at Ankara, 1957.

Haas 2003 - V. Haas, Siduri - Nahmezuli. Ein kleiner Beitrag zur Gilgameš-Forschung, Studi Micenei ed Egeo-Anatolici 45 (2003), 129-130.

Haas 2006 - V. Haas, Die hethitische Literatur: Texte, Stilistik, Motive, Berlin - New York, De Gruyter, 2006.

Keetman 2014 - J. Keetman, Der altsumerische Name /PA-GBILGA-MES/ = Gilgamě̌, Bibliotheca Orientalis 71 (2014), 30-40.

Kelly-Buccellati 2006 - M. Kelly-Buccellati, Gilgamesh at Urkesh? Literary Motifs and Iconographic Identifications, in P. Butterlin et al. (éds.), Les espaces syro-mésopotamiens - Hommage à J.C. Margueron (Subartu, 17), Turnhout, Brepols, 2006, 403-414.

Klinger 2005 - J. Klinger, Die hethitische Rezeption mesopotamischer Literatur und die Überlieferung des Gilgaměs-Epos in Hattuša, in D. Prechel (Hrsg.), Motivation und Mechanismen des Kulturkontaktes in der späten Bronzezeit (Eothen, 13), Firenze, LoGisma, 2005, 103-127.

Kraus 1980 - F.R. Kraus, Der Brief des Gilgameš, Anatolian Studies 30 (1980), 109-121. Lambert 1987 - W.G. Lambert, Gilgamesh in literature and art: the second and first mil- 
lennia, in A.E. Farkas et al. (eds.), Monster and demons in the Ancient and Medieval worlds. Papers presented in honour of Edith Porada, Mainz, von Zabern, 1987, 37-52 (ristampato in Maier 1997, $50-62$ e in Steymans Hrsg. 2010, 91-112). Laroche 1964-65 - E. Laroche, La Prière hittite: vocabulaire et typologie, École pratique des hautes études (Ve Section): Sciences Religieuses. Annuaire, 72 (1964-65), 3. 29.

Maier 1997 - J.R. Maier, Gilgamesh: a reader, Wauconda, Ill., Bolchazy-Carducci Publishers, 1997.

Matthiae 1992 - P. Matthiae, Figurative Themes and Literary Texts, in P. Fronzaroli (ed.), Literature and Literary Language at Ebla (Quaderni di Semitistica, 18), Firenze, Università - Dip. di linguistica, 1992, 219-241.

Metcalf 2015 - Ch. Metcalf, The Gods Rich in Praise. Early Greek and Mesopotamian Religious Poetry, Oxford, Oxford University Press, 2015.

Mittermayer 2010 - Ch. Mittermayer, Gilgameš im Wandel der Zeit, in Steymans Hrsg. 2010, 135-164.

Moran 1980 - W.L. Moran, Rilke and the Gilgamesh Epic, Journal of Cuneiform Studies 32 (1980), 208-210.

Nakamura 2007 - M. Nakamura, Ein bisher unbekanntes Fragment der burritischen Fassung des Gilgamě̌-Epos, in D. Groddek, M. Zorman (Hrsg.), Tabularia Hethaeorum. Hethitologische Beiträge Silvin Košak zum 65. Geburtstag (Dresdner Beiträge zur Hethitologie, 25), Wiesbaden, Harrassowitz Verlag, 2007, 557-559.

Nurullin 2012 - R. Nurullin, The Name of Gilgameš in the light of line 47 of the First Tablet of the Standard Babylonian Gilgameš Epic, Babel und Bibel 6 (2012), 209-224.

Pettinato 1991 - G. Pettinato, I Sumeri, Milano, Bompiani, 1991.

Pettinato 1992 - G. Pettinato, La saga di Gilgamesh, Milano, Rusconi, 1992.

Pogue Harrison 1992 - R. Pogue Harrison, Forests: the shadow of civilization, Chicago, The University of Chicago Press, 1992.

Rieken et al. 2009 - E. Rieken, et al., hethiter.net/: CTH 341.III, 2009 (edizione online della versione ittita del poema di Gilgamesh).

Rubio 2012 - G. Rubio, Reading Sumerian Names, II: Gilgameš, Journal of Cuneiform Studies 64 (2012), 3-16.

Sallaberger 2013 - W. Sallaberger, Das Gilgamesch-Epos. Mythos, Werk und Tradition, München, Verlag C.H. Beck, 2013.

Salvini - Wegner 2004 - M. Salvini - I. Wegner, Die mythologischen Texte (Corpus der hurritischen Sprachdenkmäler, I/6), Roma, CNR - Istituto di Studi sulle civiltà dell'Egeo e del Vicino Oriente, 2004.

Schachner 2011 - A. Schachner, Hattuscha. Auf der Suche nach dem sagenhaften Großreich der Hetbiter, München, Verlag C.H. Beck, 2011.

Steymans Hrsg. 2010 - H.U. Steymans (Hrsg.), Gilgamesch: Ikonographie eines Helden / Gilgamesh. Epic and iconography (Orbis Biblicus et Orientalis, 245), Göttingen - Fribourg, Academic Press - Vandenhoeck and Ruprecht, 2010.

Steymans 2010 - H.U. Steymans, Gilgameš im Westen, in Steymans Hrsg. 2010, 287-345. 
Thompson 1930 - R.C. Thompson, The Epic of Gilgamish, Oxford, At the Clarendon Press, 1930.

Tigay 1982 - J.H. Tigay, The evolution of the Gilgamesh Epic, Philadelphia, University of Pennsylvania Press, 1982.

Ungnad 1911 - A. Ungnad, Das Gilgamesch-Epos, Göttingen, Vandenhoeck \& Ruprecht, 1911.

van der Toorn 2007 - K. van der Toorn, Why Wisdom became a Secret: on Wisdom as a written genre, in R.J. Clifford (ed.), Wisdom Literature in Mesopotamia and Israel, Atlanta, Society of Biblical Literature, 2007, 21-29.

Waal 2014 - W. Waal, Changing fate. Hittite GUL-š-, C. Luwian GUL-zi-, H. Luwian REL-za and the Kuwanšeš-deities, in P. Taracha (ed.), Proceedings of the Eight International Congress of Hittitology, Warsaw, Agade, 2014, 1016-1034.

Waal 2015 - W. Waal, Hittite diplomatics. Studies in ancient document format and record management (Studien zu den Boğazköy-Texten, 57), Wiesbaden, Harrassowitz Verlag, 2015.

Wasserman 2011 - N. Wasserman, The distant voice of Gilgameš: the circulation and reception of the Babylonian Gilgameš Epic in ancient Mesopotamia, Archiv für Orientforschung 52 (2011), pp. 1-14.

Wilcke 1989 - C. Wilcke, Genealogical and geographical thought in the Sumerian King List, in H. Behrens et al. (eds.), DUMU-E2-DUB-BA-A. Studies in honor of Ake W. Sjöberg, Philadelphia, The Samuel Noah Kramer Fund / The University Museum, 1989, 557-571.

Yakubovich 2014 - I. Yakubovich, The Luwian deity Kwanza, Aramazd 8 (2014), 282 297.

Ziolkowski 2011 - Th. Ziolkowski, Gilgamesh Among Us: Modern Encounters with the Ancient Epic, Ithaca, NY, Cornell University Press, 2011. 